\title{
THE FOURTH MOMENT THEOREM ON THE POISSON SPACE ${ }^{1}$
}

\author{
By Christian Döbler and Giovanni PecCati
}

\section{Université du Luxembourg}

\begin{abstract}
We prove a fourth moment bound without remainder for the normal approximation of random variables belonging to the Wiener chaos of a general Poisson random measure. Such a result - that has been elusive for several years-shows that the so-called 'fourth moment phenomenon', first discovered by Nualart and Peccati [Ann. Probab. 33 (2005) 177-193] in the context of Gaussian fields, also systematically emerges in a Poisson framework. Our main findings are based on Stein's method, Malliavin calculus and Mecketype formulae, as well as on a methodological breakthrough, consisting in the use of carré-du-champ operators on the Poisson space for controlling residual terms associated with add-one cost operators. Our approach can be regarded as a successful application of Markov generator techniques to probabilistic approximations in a nondiffusive framework: as such, it represents a significant extension of the seminal contributions by Ledoux [Ann. Probab. 40 (2012) 2439-2459] and Azmoodeh, Campese and Poly [J. Funct. Anal. 266 (2014) 2341-2359]. To demonstrate the flexibility of our results, we also provide some novel bounds for the Gamma approximation of nonlinear functionals of a Poisson measure.
\end{abstract}

\section{Introduction.}

1.1. Overview. The aim of this paper is to prove a fourth moment bound without remainder for the normal approximation of random variables belonging to the Wiener chaos of a general Poisson measure. Differently from previous fourth moment limit theorems on the Poisson space proved in the literature, our main findings do not require that the involved random variables have the form of multiple integrals with a kernel of constant sign [see Eichelsbacher and Thäle (2014), Lachièze-Rey and Peccati (2013), Schulte (2016)], nor that they are finite homogeneous sums [see Peccati and Zheng (2014)] or that they belong to Wiener chaoses of lower orders [see Bourguin and Peccati (2016), Peccati and Taqqu (2008)]. As discussed below, the methodological breakthrough yielding such an achievement, consists in the use of carré-du-champ operators on the Poisson space, that we shall systematically exploit in connection with Mecke-type formulae and Stein's method

Received January 2017; revised July 2017.

${ }^{1}$ Supported by the grant F1RMTH-PUL-15STAR (STARS) at Luxembourg University.

MSC2010 subject classifications. Primary 60F05; secondary 60H07, $60 \mathrm{H} 05$.

Key words and phrases. Poisson functionals, multiple Wiener-Itô integrals, fourth moment theorem, carré-du-champ operator, Berry-Esseen bounds, Gaussian approximation, Gamma approximation, Malliavin calculus, Stein's method. 
[see Chen, Goldstein and Shao (2011), Nourdin and Peccati (2012)]. We will see that using carré-du-champ operators instead of norms of Malliavin derivatives [as done in the already quoted references Bourguin and Peccati (2016), Eichelsbacher and Thäle (2014), Lachièze-Rey and Peccati (2013), Peccati and Taqqu (2008), Peccati and Zheng (2014), Schulte (2016)] will allow us to bypass at once almost all combinatorial difficulties - in particular connected to multiplication formulae on configuration spaces - that have systematically marred previous attempts.

We stress that the idea of using carré-du-champ operators, in order to deduce quantitative limit theorems by means of Stein's method, originates in the groundbreaking works Azmoodeh, Campese and Poly (2014), Azmoodeh et al. (2016), Ledoux (2012), where the authors apply the powerful techniques of Gamma calculus in the framework of eigenspaces of diffusive Markov generators [see Bakry, Gentil and Ledoux (2014) for definitions, as well as Chen and Poly (2015) for an introduction to this approach]. As demonstrated in Section 3, our results show that such an approach can be fruitfully applied and extended, in order to control residual terms arising from the application of Stein's method in a nondiffusive context.

1.2. Further historical details. The so-called fourth moment phenomenon was first discovered in Nualart and Peccati (2005), where the authors proved that a sequence of normalized random variables, belonging to a fixed Wiener chaos of a Gaussian field, converge in distribution to a Gaussian random variable if and only if their fourth cumulant converges to zero. Such a result constitutes a dramatic simplification of the method of moments and cumulants [see, e.g., Nourdin and Peccati (2012), page 202], and represents a rough infinite-dimensional counterpart of classical results by de Jong; see de Jong (1987, 1989, 1990), as well as Döbler and Peccati (2017a), Döbler and Peccati (2017b) for recent advances. A particularly fruitful line of research was initiated in Nourdin and Peccati (2009a), where it is proved that the results of Nualart and Peccati (2005) can be recovered from very general estimates, obtained by combining the Malliavin calculus of variations with Stein's method for normal approximation. Precisely, one remarkable achievement of this approach is the bound

$$
d_{\mathrm{Kol}}(F, N) \leq \sqrt{\frac{q-1}{3 q}\left(\mathbb{E}\left[F^{4}\right]-3\right)},
$$

where $d_{\text {Kol }}$ stands for the Kolmogorov distance between the laws of two random variables, $F$ is a normalized multiple Wiener-Itô integral of order $q \geq 1$ on a Gaussian space and $N$ denotes a standard normal random variable [see, e.g., Theorem 5.2.6 in Nourdin and Peccati (2012), where analogous bounds for other metrics are also stated]. Such a discovery has been the seed of a fruitful stream of research, now consisting of several hundred papers, where the results of Nualart and Peccati $(2005,2009 a)$ have been extended and applied to a variety of frameworks, ranging from free probability to stochastic geometry, compressed sensing and 
time-series analysis; see the webpage https://sites.google.com/site/malliavinstein/ home for a constantly updated list, as well as the monograph Nourdin and Peccati (2012) and the reference Ledoux, Nourdin and Peccati (2015) for recent developments related to functional inequalities.

The line of research pursued in the present work stems from the two papers Peccati and Zheng (2010), Peccati et al. (2010), where the authors adapted the techniques introduced in Nourdin and Peccati (2009a) to the framework of nonlinear Poisson functionals, in particular by combining Stein's method with a discrete version of Malliavin calculus on configuration spaces. As anticipated, the principal aim of this work is to positively answer the following question:

Can one prove a bound comparable to (1) on the Poisson space?

Such a question has stayed open since the publication of Peccati et al. (2010) and, so far, answers have only been found in very special cases; see Remark 1.5 below.

One should notice that the relevance of the techniques developed in Peccati and Zheng (2010), Peccati et al. (2010) has been greatly amplified by the pathbreaking reference Reitzner and Schulte (2013), where it is shown that one can use Malliavin-Stein techniques on the Poisson space in order to study the fluctuation of random objects arising in the context of random geometric structures on configuration spaces like, for example, random graphs or random tessellations. Such a connection with stochastic geometry has generated a remarkable body of work that has recently culminated in the publication of the monograph Peccati and Reitzner (2016). The reader is referred to Lachièze-Rey, Schulte and Yukich (2016), Last, Peccati and Schulte (2016) for recent developments connected to Mehler formulae, stabilization and second-order Poincaré inequalities, and to Bachmann and Peccati (2016) for some related concentration estimates in a geometric context.

1.3. Main results for normal approximations. We fix an arbitrary measurable space $(\mathcal{Z}, \mathscr{Z})$ endowed with a $\sigma$-finite measure $\mu$. Furthermore, we let

$$
\mathscr{Z}_{\mu}:=\{B \in \mathscr{Z}: \mu(B)<\infty\}
$$

and denote by

$$
\eta=\{\eta(B): B \in \mathscr{Z}\}
$$

a Poisson measure on $(\mathcal{Z}, \mathscr{Z})$ with control $\mu$, defined on a suitable probability space $(\Omega, \mathcal{F}, \mathbb{P})$. We recall that the distribution of $\eta$ is completely determined by the following two facts: (i) for each finite sequence $B_{1}, \ldots, B_{m} \in \mathscr{Z}$ of pairwise disjoint sets, the random variables $\eta\left(B_{1}\right), \ldots, \eta\left(B_{m}\right)$ are independent and (ii) that for every $B \in \mathscr{Z}$, the random variable $\eta(B)$ has the Poisson distribution with mean $\mu(B)$. Here, we have extended the family of Poisson distributions to the parameter region $[0,+\infty]$ in the usual way. For $B \in \mathscr{Z}_{\mu}$, we also write $\hat{\eta}(B):=\eta(B)-\mu(B)$ and denote by

$$
\hat{\eta}=\left\{\hat{\eta}(B): B \in \mathscr{Z}_{\mu}\right\}
$$


the compensated Poisson measure associated with $\eta$. As discussed in Section 2.1, we require throughout the paper that $\eta$ is proper, that is, that $\eta$ can be a.s. represented as a (possibly infinite) random sum of Dirac masses. Without loss of generality, we may and will assume that $\mathcal{F}=\sigma(\eta)$. In order to state our main results, we introduce the following fundamental objects from stochastic analysis on the Poisson space. For precise definitions and further explanation, we refer to Peccati and Reitzner (2016), in particular to its first chapter Last (2016), as well as to Last and Penrose (2017) and Section 2. For a nonnegative integer $q$ and a square-integrable kernel function $f \in L^{2}\left(\mu^{q}\right)$, we denote by $I_{q}(f)$ the $q$ th order multiple Wiener-Itô integral of $f$ with respect to $\hat{\eta}$. If $L$ denotes the generator of the Ornstein-Uhlenbeck semigroup with respect to $\eta$, then it is well known that $-L$ has pure point spectrum given by the set of nonnegative integers and that, for $q \in \mathbb{N}_{0}=\{0,1,2, \ldots\}, F$ is an eigenfunction of $-L$ with eigenvalue $q$, if and only if $F=I_{q}(f)$ for some $f \in L^{2}\left(\mu^{q}\right)$. The corresponding eigenspace $C_{q}$ is called the qth Wiener chaos associated with $\eta$.

Next, we introduce the probabilistic distances in which our bounds are expressed. For $m \in \mathbb{N}$, denote by $\mathcal{H}_{m}$ the class of those $(m-1)$-times differentiable test functions $h$ on $\mathbb{R}$ such that $h^{(m-1)}$ is Lipschitz-continuous and we have

$$
\left\|h^{(l)}\right\|_{\infty} \leq 1 \quad \text { for } l=1, \ldots, m .
$$

Here and elsewhere, for an arbitrary function $g$ on $\mathbb{R}$, we use the notation

$$
\left\|g^{\prime}\right\|_{\infty}:=\sup _{x \neq y} \frac{|g(y)-g(x)|}{|y-x|} \in[0,+\infty]
$$

for the minimum Lipschitz-constant of $g$. This does not cause any confusion because this quantity coincides with the supremum norm of the derivative $g^{\prime}$ of $g$ when $g$ happens to be differentiable. For real random variables $X$ and $Y$ such that $\mathbb{E}|X|, \mathbb{E}|Y|<\infty$, we denote by

$$
d_{m}(X, Y):=d_{m}(\mathcal{L}(X), \mathcal{L}(Y)):=\sup _{h \in \mathcal{H}_{m}}|\mathbb{E}[h(X)]-\mathbb{E}[h(Y)]|
$$

the distance between the distributions of $X$ and $Y$ induced by the class $\mathcal{H}_{m}$; observe that $d_{1}$ coincides with the classical 1-Wasserstein distance; see, for example, Nourdin and Peccati (2012), Appendix C, and the references therein. We will also study the Kolmogorov distance between the laws of $X$ and $Y$, given by

$$
d_{\mathrm{Kol}}(X, Y):=\sup _{x \in \mathbb{R}}|\mathbb{P}[X \leq x]-\mathbb{P}[Y \leq x]| .
$$

It is a well-known fact [see, e.g., Nourdin and Peccati (2012), Appendix C, and the references therein] that if $X$ is a generic random variable and $Y$ has a density bounded by $c \in(0, \infty)$, then

$$
d_{\mathrm{Kol}}(X, Y) \leq \sqrt{2 c d_{1}(X, Y)} .
$$


The assumptions in our main results will be expressed in terms of the add-one cost operator $D^{+}$that is defined as follows: if $F=\mathfrak{f}(\eta)$ is a functional of $\eta$, then

$$
D_{z}^{+} F:=\mathfrak{f}\left(\eta+\delta_{z}\right)-\mathfrak{f}(\eta), \quad z \in \mathcal{Z},
$$

in such a way that $D^{+} F$ can be regarded as a random function with domain equal to $\mathcal{Z}$. See Section 2.1 for a formal discussion of such an object.

DEFINITION 1.1. Let $F$ be an $\mathcal{F}$-measurable random variable [recall that $\mathcal{F}=$ $\sigma(\eta)]$.

(i) We say that $F$ satisfies Assumption A if $F \in L^{4}(\mathbb{P})$ and if the four random functions $D^{+} F, F D^{+} F,\left(D^{+} F\right)^{4}$ and $F^{3} D^{+} F$ are all elements of the space $L^{1}(\Omega \times Z, \mathcal{F} \otimes \mathscr{Z}, \mathbb{P} \otimes \mu)=: L^{1}(\mathbb{P} \otimes \mu)$.

(ii) We say that $F$ satisfies Assumption $A^{\text {loc }}$ if there exists a set $Z_{0} \in \mathscr{Z}$ with the following properties: (a) $\mu\left(\mathcal{Z} \backslash Z_{0}\right)=0$, and (b) for every fixed $z \in Z_{0}$, the random variable $D_{z}^{+} F$ verifies Assumption A.

REMARK 1.2. (a) Requiring that a given functionals satisfies Assumption A or Assumption $\mathrm{A}^{\text {loc }}$ is necessary in this paper, in order for us to apply Mecketype identities [see (12)-(13) below], as well as to exploit several almost sure representations of Malliavin and carré-du-champ operators. Both assumptions seem therefore to be (rather minimal) artefacts of the specific techniques adopted in the present paper, that are bound to be removed by further progress in the field. See Döbler, Vidotto and Zheng (2017) for several recent advances in this direction, partially building on the findings of the present paper, showing how Assumption A can be avoided in the normal approximation of chaotic random variables in the Wasserstein distance, by using an adequate version of the exchangeable pairs approach of Stein's method. We also notice that a bound like (6) on the Kolmogorov distance (which is of the same order as the corresponding bound on the Wasserstein distance), is for the time being outside the scope of exchangeable pairs.

(b) Using, for example, the multiplication formula stated in Last (2016), Proposition 5, one can easily prove that both Assumption A and Assumption $\mathrm{A}^{\text {loc }}$ are verified, whenever $F$ has the form

$$
F=\sum_{q=0}^{M} I_{q}\left(f_{q}\right),
$$

where $M<\infty$ and each $f_{q}$ is bounded and such that its support is contained in a rectangle of the type $C \times \cdots \times C$, where $C \in \mathscr{Z}$ verifies $\mu(C)<\infty$. Such a class of random variables contains most $U$-statistics that are relevant for geometric applications [see the surveys Lachièze-Rey and Reitzner (2016), Schulte and Thäle (2016) and the references therein], as well as nonlinear functionals of Volterra Lévy processes Peccati and Zheng (2010), Peccati et al. (2010), and the 
finite homogeneous sums in independent Poisson random variables considered in Peccati and Zheng (2014). A similar remark applies to the assumptions appearing in the statement of our main abstract bounds in Proposition 4.1 and Proposition 4.3.

The next result is the main finding of the paper: it provides quantitative fourth moment estimates with completely explicit constants, both in the 1-Wasserstein and Kolmogorov distances, for random variables living in the Wiener chaos of a Poisson measure. Remarkably, the order of the bound (as a function of the fourth cumulant $\left.\mathbb{E}\left[F^{4}\right]-3\right)$ is the same for the two distances, thus significantly improving the estimate on $d_{\mathrm{Kol}}$ that one could deduce from (2).

THEOREM 1.3 (Fourth moment bounds on the Poisson space). Fix an integer $q \geq 1$ and let $F=I_{q}(f)$ be a multiple Wiener-Itô integral with respect to $\hat{\eta}$. Assume that $F$ verifies Assumption A and that $\mathbb{E}\left[F^{2}\right]=1$; denote by $N \sim \mathcal{N}(0,1)$ a standard normal random variable. Then

$$
\begin{aligned}
d_{1}(F, N) & \leq\left(\sqrt{\frac{2}{\pi}} \frac{2 q-1}{2 q}+\frac{\sqrt{4 q-3}}{\sqrt{q}}\right) \sqrt{\mathbb{E}\left[F^{4}\right]-3} \\
& \leq\left(\sqrt{\frac{2}{\pi}}+2\right) \sqrt{\mathbb{E}\left[F^{4}\right]-3}
\end{aligned}
$$

(in the above situation one automatically has that $\mathbb{E}\left[F^{4}\right] \geq 3$ ). Moreover, if, in addition to Assumption A, $F$ also satisfies Assumption $\mathrm{A}^{\text {loc }}$, then

$$
\begin{aligned}
& d_{\mathrm{Kol}}(F, N) \leq\left(11+2^{3 / 2}\left(\mathbb{E}\left[F^{4}\right]^{1 / 2}+\mathbb{E}\left[F^{4}\right]^{1 / 4}\right)\right) \sqrt{\mathbb{E}\left[F^{4}\right]-3} \text { and } \\
& d_{\mathrm{Kol}}(F, N) \leq 15.6 \sqrt{\mathbb{E}\left[F^{4}\right]-3} .
\end{aligned}
$$

The following result is an immediate consequence of the bound (4).

COROLlaRY 1.4 (Fourth moment theorem on the Poisson space). For each $n \in \mathbb{N}$ let $q_{n} \geq 1$ be an integer and let $F_{n}=I_{q_{n}}\left(f_{n}\right)$ be a multiple Wiener-Ito integral of some symmetric kernel $f_{n} \in L^{2}\left(\mu^{q_{n}}\right)$ such that

$$
\lim _{n \rightarrow \infty} \mathbb{E}\left[F_{n}^{2}\right]=\lim _{n \rightarrow \infty} q_{n} !\left\|f_{n}\right\|_{2}^{2}=1 \text { and } \lim _{n \rightarrow \infty} \mathbb{E}\left[F_{n}^{4}\right]=3 \text {. }
$$

Then, if each $F_{n}$ satisfies Assumption A, the sequence $\left(F_{n}\right)_{n \in \mathbb{N}}$ converges in distribution to a standard normal random variable $N$ in the sense of the 1-Wasserstein distance.

REMARK 1.5. (a) As mentioned before, so far, the fourth moment theorem on the Poisson space has only been known in very special cases: for double integrals, that is, for $q=2$, the qualitative fourth moment theorem was proved in 
Peccati and Taqqu (2008). Under different assumptions, this result is also proved in Bourguin and Peccati (2016) where also a qualitative fourth moment theorem for $q=3$ is derived. We would like to mention that the method of proof applied in Bourguin and Peccati (2016) is rather ad hoc and cannot be generalized to higher values of $q$. We also stress that all existing quantitative fourth moment theorems on the Poisson space make the restrictive assumption that the kernel function $f$ has a constant sign [see, e.g., Eichelsbacher and Thäle (2014), Lachièze-Rey and Peccati (2013), Schulte (2016)]. Furthermore, the multiplicative constants in these results depend on the order $q$ in a nonexplicit way, implying that a statement in the spirit of Corollary 1.4 cannot be inferred from them. We also mention Peccati and Zheng (2014), where one can find a fourth moment theorem for sequences of chaotic elements having the form of homogeneous sums in independent Poisson random variables whose variance is bounded away from zero, as well as Bourguin and Peccati (2014), where the authors prove a fourth moment theorem for multiple integrals with respect of a noncommutative Poisson measure (in the framework of free probability theory), under an additional tameness assumption. Finally, the already quoted recent contribution Döbler, Vidotto and Zheng (2017) also contains several multidimensional extensions of the main findings of the present work.

(b) We find it quite remarkable that our fourth moment bounds do not require any additional error term accounting for the discreteness of the Poisson space as is necessary, for example, in the context of degenerate $U$-statistics [see, e.g., de Jong (1990) and Döbler and Peccati (2017a)] as well as for discrete multiple integrals of independent Rademacher random variables [see Döbler and Krokowski (2017)] where such fourth moment theorems without remainder do not hold.

We also notice the following negative result.

Proposition 1.6. For each $q \in \mathbb{N}$, there exists no Gaussian random variable with positive variance in the qth Wiener chaos $C_{q}$ associated with $\eta$.

1.4. Main results on Gamma approximations. For $v>0$, we denote by $\bar{\Gamma}(v)$ the so-called centered Gamma distribution with parameter $v$ which by definition is the distribution of

$$
Z_{v}:=2 X_{v / 2,1}-v
$$

where $X_{v / 2,1}$ has the usual Gamma distribution on $[0,+\infty)$ with shape parameter $\nu / 2$ and rate 1 . In particular, one has

$$
\mathbb{E}\left[Z_{v}\right]=0 \quad \text { and } \quad \operatorname{Var}\left(Z_{v}\right)=\mathbb{E}\left[Z_{v}^{2}\right]=2 v
$$

Moreover, the following moment identity [already exploited in Nourdin and Peccati (2009b)] will play an important role in what follows:

$$
\mathbb{E}\left[Z_{v}^{4}\right]-12 \mathbb{E}\left[Z_{v}^{3}\right]-12 v^{2}+48 v=0
$$

The next result is the counterpart of Theorem 1.3 for centered Gamma approximation. 
THEOREM 1.7 (Fourth moment bound for Gamma approximation). Fix $v>0$ as well as an integer $q \geq 1$ and let $F=I_{q}(f)$ be a multiple Wiener-Itô integral with respect to $\hat{\eta}$, verifying Assumption $\mathrm{A}$. Assume that $F \in L^{4}(\mathbb{P})$ and that $\mathbb{E}\left[F^{2}\right]=2 v$. Also, let $Z_{v} \sim \bar{\Gamma}(v)$ have the centered Gamma distribution with parameter $v$. Then we have the following bound:

$$
\begin{aligned}
d_{2}\left(F, Z_{v}\right) \leq & C_{1}(v) \sqrt{\left|\mathbb{E}\left[F^{4}\right]-12 \mathbb{E}\left[F^{3}\right]-12 v^{2}+48 v\right|} \\
& +C_{2}(v)\left(\frac{1}{q} \int_{\mathcal{Z}} \mathbb{E}\left[\left|D_{z}^{+} F\right|^{4}\right] \mu(d z)\right)^{1 / 2},
\end{aligned}
$$

where $D^{+}$denotes the add-one-cost operator associated with $\eta$ (see Section 2) and where we can let

$$
\begin{aligned}
& C_{1}(v):=\frac{1}{\sqrt{3}} \max \left(1, \frac{2}{v}\right) \text { and } \\
& C_{2}(v):=\frac{1}{\sqrt{6}} \max \left(1, \frac{2}{v}\right)+\max \left(\sqrt{2 v}, \sqrt{\frac{2}{v}}+\sqrt{\frac{v}{2}}\right) .
\end{aligned}
$$

REMARK 1.8. (a) The bound (8) displays an additional term, not directly connected to moments, that is not present in the estimate (3) for normal approximations. For the time being, it is a challenging open problem to determine whether such a term can be removed.

(b) The previous result implies that, if, for $n \in \mathbb{N}, F_{n} \in \operatorname{Ker}\left(L+q_{n} I\right)\left(q_{n} \geq 1\right)$ is a sequence of random variables verifying Assumption $\mathrm{A}$ and such that

$$
\lim _{n \rightarrow \infty} \mathbb{E}\left[F_{n}^{2}\right]=2 v, \quad \lim _{n \rightarrow \infty}\left(\mathbb{E}\left[F_{n}^{4}\right]-12 \mathbb{E}\left[F_{n}^{3}\right]\right)=12 v^{2}-48 v
$$

and

$$
\lim _{n \rightarrow \infty} \int_{\mathcal{Z}} \mathbb{E}\left[\left|D_{z}^{+} F_{n}\right|^{4}\right] \mu(d z)=0,
$$

then $F_{n}$ converges in distribution to $Z_{\nu}$. This result largely extends the Gamma limit theorem for double integrals stated in Peccati and Thäle (2013), Proposition 4.7, where (9) is replaced by the requirement that $f_{n} \rightarrow 0$ in $L^{4}$, with $f_{n}$ denoting the function in $q_{n}$ variables such that $F_{n}=I_{q_{n}}\left(f_{n}\right)$. In general, if $q_{n} \equiv q$, and $F_{n}$ has the form $I_{q}\left(f_{n}\right)$ for some sufficiently regular kernel $f_{n}$, then one sufficient condition in order to have (9) is that all contractions of the type $f_{n} \star_{b}^{a} f_{n}$ with $a<b$ converge to zero in $L^{2}$, where the definition of $f_{n} \star_{b}^{a} f_{n}$ can be found, for example, in Last (2016), Section 6; see the computations contained in Peccati et al. (2010), pages 465-466. A detailed discussion of the Gamma bound (8) via the use of contraction operators [in the sprit, for example, of Fissler and Thäle (2016), Peccati and Thäle (2013)] seems to be outside the scope of the present work, and will be tackled elsewhere; see also Döbler and Peccati (2017b). 
(c) The parametrization of the Gamma distribution which we use is mainly chosen for historical reasons [see, e.g., the papers Nourdin and Peccati (2009b), Peccati and Thäle (2013)] and for convenience because multiple integrals are naturally centered. Of course, in general, the Gamma distribution also involves an unimportant rate parameter $\lambda>0$. This situation can be easily dealt with by considering $\lambda^{-1} Z_{v}$ in place of $Z_{v}$ and by using the simple inequality $d_{2}\left(F, \lambda^{-1} Z_{v}\right) \leq$ $\lambda^{-1} d_{2}\left(\lambda F, Z_{\nu}\right)$ which can be bounded by means of (8) whenever $F$ is in $C_{q}$ and $\mathbb{E}\left[F^{2}\right]=2 \nu \lambda^{-2}$.

1.5. Plan. The paper is organized as follows. Section 2 contains preliminary results concerning stochastic analysis on the Poisson space. Section 3 focusses on several new estimates for multiple integrals, whereas Section 4 and Section 5 deal with the proofs of our main results. Finally, Section 6 contains the proofs of some technical lemmas.

2. Elements of stochastic analysis on the Poisson space. In this section, we describe our theoretical framework in more detail, by adopting the language of Last (2016), corresponding to Chapter 1 in Peccati and Reitzner (2016). See also the monograph Last and Penrose (2017).

2.1. Setup. In what follows, we will view the Poisson process $\eta$ as a random element taking values in the space $\mathbf{N}_{\sigma}=\mathbf{N}_{\sigma}(\mathcal{Z})$ of all $\sigma$-finite point measures $\chi$ on $(\mathcal{Z}, \mathscr{Z})$ that satisfy $\chi(B) \in \mathbb{N}_{0} \cup\{+\infty\}$ for all $B \in \mathscr{Z}$. Such a space is equipped with the smallest $\sigma$-field $\mathscr{N}_{\sigma}:=\mathscr{N}_{\sigma}(\mathcal{Z})$ such that, for each $B \in \mathscr{Z}$, the mapping $\mathbf{N}_{\sigma} \ni \chi \mapsto \chi(B) \in[0,+\infty]$ is measurable. As anticipated, throughout the paper we shall assume that the process $\eta$ is proper, in the sense that $\eta$ can be $\mathbb{P}$-a.s. represented in the form

$$
\eta=\sum_{n=1}^{\eta(\mathcal{Z})} \delta_{X_{n}}
$$

where $\left\{X_{n}: n \geq 1\right\}$ denotes a countable collection of random elements with values in $\mathcal{Z}$ and where, for $z \in \mathcal{Z}$, we write $\delta_{z}$ for the Dirac measure at $z$. A sufficient condition for $\eta$ to be proper is, for example, that $(\mathcal{Z}, \mathscr{Z})$ is a Polish space endowed with its Borel $\sigma$-field, with $\mu$ being $\sigma$-finite as above. See Last and Penrose (2017), Section 6.1, and Last (2016), pages 2-3, for more details. Furthermore, Corollary 3.7 in Last and Penrose (2017) states that for each Poisson process $\eta$, there exists (maybe on a different probability space) a proper Poisson process $\eta^{*}$ which has the same distribution as $\eta$. Since all our results depend uniquely on the distribution of $\eta$, it is no restriction of generality to assume that $\eta$ is proper.

Now denote by $\mathbf{F}\left(\mathbf{N}_{\sigma}\right)$ the class of all measurable functions $\mathfrak{f}: \mathbf{N}_{\sigma} \rightarrow \mathbb{R}$ and by $\mathcal{L}^{0}(\Omega):=\mathcal{L}^{0}(\Omega, \mathcal{F})$ the class of real-valued, measurable functions $F$ on $\Omega$. Note that, as $\mathcal{F}=\sigma(\eta)$, each $F \in \mathcal{L}^{0}(\Omega)$ can be written as $F=\mathfrak{f}(\eta)$ for some 
measurable function $\mathfrak{f}$. This $\mathfrak{f}$, called a representative of $F$, is $\mathbb{P}_{\eta}$-a.s. uniquely defined, where $\mathbb{P}_{\eta}=\mathbb{P} \circ \eta^{-1}$ is the image measure of $\mathbb{P}$ under $\eta$ on the space $\left(\mathbf{N}_{\sigma}, \mathscr{N}_{\sigma}\right)$.

Using a representative $\mathfrak{f}$ of $F$, we can define the so-called add-one cost operator $D^{+}=\left(D_{z}^{+}\right)_{z \in \mathcal{Z}}$ on $\mathcal{L}^{0}(\Omega)$ [recall that we assume $\mathcal{F}=\sigma(\eta)$ ] by

$$
D_{z}^{+} F:=\mathfrak{f}\left(\eta+\delta_{z}\right)-\mathfrak{f}(\eta), \quad z \in \mathcal{Z}
$$

similarly, we define $D^{-}$on $\mathcal{L}^{0}(\Omega)$ via

$$
\begin{aligned}
& D_{z}^{-} F:=\mathfrak{f}(\eta)-\mathfrak{f}\left(\eta-\delta_{z}\right) \quad \text { if } z \in \operatorname{supp}(\eta) \quad \text { and } \\
& D_{z}^{-} F:=0 \quad \text { otherwise, }
\end{aligned}
$$

where here

$$
\operatorname{supp}(\chi):=\{z \in \mathcal{Z}: \text { for all } A \in \mathscr{Z} \text { s.t. } z \in A: \chi(A) \geq 1\}
$$

stands for the support of the measure $\chi \in \mathbf{N}_{\sigma}$; note that, since $\eta$ is proper, if $z \in$ $\operatorname{supp}(\eta)$, then $\eta-\delta_{z} \in \mathbf{N}_{\sigma}$. Intuitively, $-D^{-}$is a remove-one cost operator. We stress that the definitions of $D^{+} F$ and $D^{-} F$ are, respectively, $\mathbb{P} \otimes \mu$-a.e. and $\mathbb{P}$ a.s. independent of the choice of the representative $\mathfrak{f}$; see, for example, Last and Penrose (2011) for the case of $D^{+}$, whereas the case of $D^{-}$can be dealt with by using the Mecke formula (13) below, Lemma 2.4. Similarly, the conditions stated in Assumption A and Assumption $\mathrm{A}^{\text {loc }}$ do not depend on the choice of the representative $\mathfrak{f}$.

We conclude the section by observing that the operator $D^{+}$can be canonically iterated by setting $D^{(1)}:=D^{+}$and, for $n \geq 2$ and $z_{1}, \ldots, z_{n} \in \mathcal{Z}$ and $F \in \mathcal{L}^{0}(\Omega)$, by recursively defining

$$
D_{z_{1}, \ldots, z_{n}}^{(n)} F:=D_{z_{1}}^{+}\left(D_{z_{2}, \ldots, z_{n}}^{(n-1)} F\right)
$$

2.2. $L^{1}$ theory: Mecke formula and $\Gamma_{0}$. A central formula in the theory of Poisson processes is the so-called Mecke formula from Mecke (1967) which says that for each measurable function $h: \mathbf{N}_{\sigma} \times \mathcal{Z} \rightarrow[0,+\infty]$ the identity

$$
\mathbb{E}\left[\int_{\mathcal{Z}} h\left(\eta+\delta_{z}, z\right) \mu(d z)\right]=\mathbb{E}\left[\int_{\mathcal{Z}} h(\eta, z) \eta(d z)\right]
$$

holds true; see Last and Penrose (2017), Chapter 4, for a modern discussion of this fundamental result. We will pervasively use the following consequence of (12). 
LEMMA 2.1. For some integer $d \geq 1$, let $\mathfrak{f}_{1}, \ldots, \mathfrak{f}_{d}$ be measurable mappings from $\mathbf{N}_{\sigma}$ into $[0,+\infty]$, and let $V:[0,+\infty]^{2 d} \rightarrow[0,+\infty]$ be measurable. Then

$$
\begin{aligned}
\mathbb{E}\left[\int_{\mathcal{Z}} V(z) \mu(d z)\right] \\
:=\mathbb{E}\left[\int_{\mathcal{Z}} V\left(\mathfrak{f}_{1}(\eta), \mathfrak{f}_{1}\left(\eta+\delta_{z}\right), \ldots, \mathfrak{f}_{d}(\eta), \mathfrak{f}_{d}\left(\eta+\delta_{z}\right)\right) \mu(d z)\right] \\
=\mathbb{E}\left[\int_{\mathcal{Z}} V\left(\mathfrak{f}_{1}\left(\eta-\delta_{z}\right), \mathfrak{f}_{1}(\eta), \ldots, \mathfrak{f}_{d}\left(\eta-\delta_{z}\right), \mathfrak{f}_{d}(\eta)\right) \eta(d z)\right] .
\end{aligned}
$$

Both sides of (13) do not change if any of the $\mathfrak{f}_{i}, i=1, \ldots, d$ is replaced with another measurable mapping $\widehat{\mathfrak{f}}_{i}$ such that $\mathfrak{f}_{i}=\widehat{\mathfrak{f}}_{i}$, a.s. $-\mathbb{P}_{\eta}$.

PROOF. Apply relation (12) to the random function

$$
\begin{aligned}
h\left(\eta+\delta_{z}, z\right):= & V(z)=V\left(\mathfrak{f}_{1}(\eta), \mathfrak{f}_{1}\left(\eta+\delta_{z}\right), \ldots, \mathfrak{f}_{d}(\eta), \mathfrak{f}_{d}\left(\eta+\delta_{z}\right)\right) \\
= & V\left(\mathfrak{f}_{1}\left(\eta+\delta_{z}-\delta_{z}\right), \mathfrak{f}_{1}\left(\eta+\delta_{z}\right), \ldots, \mathfrak{f}_{d}\left(\eta+\delta_{z}-\delta_{z}\right), \mathfrak{f}_{d}\left(\eta+\delta_{z}\right)\right) \\
& \cdot \mathbf{1}_{\left\{\left(\eta+\delta_{z}\right)(\{z\}) \geq 1\right\},}
\end{aligned}
$$

in such a way that

$$
h(\eta, z)=V\left(\mathfrak{f}_{1}\left(\eta-\delta_{z}\right), \mathfrak{f}_{1}(\eta), \ldots, \mathfrak{f}_{d}\left(\eta-\delta_{z}\right), \mathfrak{f}_{d}(\eta)\right) \mathbf{1}_{\{\eta(\{z\}) \geq 1\}} .
$$

The last sentence in the statement follows from Last and Penrose (2011), Lemma 2.4.

REMARK 2.2. Plainly, formulae (12) and (13) continue to hold when the functions $h\left(\eta+\delta_{z}, z\right)$ and $V(z)$ are in $L^{1}(\mathbb{P} \otimes \mu)$, without necessarily having a constant sign.

For random variables $F, G \in \mathcal{L}^{0}(\Omega)$ such that $D^{+} F D^{+} G \in L^{1}(\mathbb{P} \otimes \mu)$, we define

$$
\Gamma_{0}(F, G):=\frac{1}{2}\left\{\int_{\mathcal{Z}}\left(D_{z}^{+} F D_{z}^{+} G\right) \mu(d z)+\int_{\mathcal{Z}}\left(D_{z}^{-} F D_{z}^{-} G\right) \eta(d z)\right\}
$$

which verifies $\mathbb{E}\left[\left|\Gamma_{0}(F, G)\right|\right]<\infty$, and $\mathbb{E}\left[\Gamma_{0}(F, G)\right]=\mathbb{E}\left[\int_{\mathcal{Z}}\left(D_{z}^{+} F D_{z}^{+} G\right) \mu(d z)\right]$, in view of the Mecke formula (13). The following statement will play a fundamental role in our work.

LEMMA 2.3 ( $L^{1}$ integration by parts). Let $G, H \in \mathcal{L}^{0}(\Omega)$ be such that

$$
G D^{+} H, D^{+} G D^{+} H \in L^{1}(\mathbb{P} \otimes \mu) \text {. }
$$

Then

$$
\mathbb{E}\left[G\left(\int_{\mathcal{Z}} D_{z}^{+} H \mu(d z)-\int_{\mathcal{Z}} D_{z}^{-} H \eta(d z)\right)\right]=-\mathbb{E}\left[\Gamma_{0}(G, H)\right] .
$$


PRoof. The assumptions in the statement imply that $\left(G+D^{+} G\right) D^{+} H \in$ $L^{1}(\mathbb{P} \otimes \mu)$. Applying (13) and Remark 2.2 to

$$
V(z)=\mathfrak{g}\left(\eta+\delta_{z}\right)\left\{\mathfrak{h}\left(\eta+\delta_{z}\right)-\mathfrak{h}(\eta)\right\},
$$

where $\mathfrak{g}$ and $\mathfrak{h}$ are representatives of $G$ and $H$, respectively, yields that

$$
\mathbb{E}\left[G \int_{\mathcal{Z}} D_{z}^{-} H \eta(d z)\right]=\mathbb{E}\left[\int_{\mathcal{Z}}\left(G+D_{z}^{+} G\right) D_{z}^{+} H \mu(d z)\right],
$$

which gives immediately the desired conclusion.

2.3. $L^{2}$ theory, part 1: Multiple integrals. For an integer $p \geq 1$, we denote by $L^{2}\left(\mu^{p}\right)$ the Hilbert space of all square-integrable and real-valued functions on $\mathcal{Z}^{p}$ and we write $L_{s}^{2}\left(\mu^{p}\right)$ for the subspace of those functions in $L^{2}\left(\mu^{p}\right)$ which are $\mu^{p}$-a.e. symmetric. Moreover, for ease of notation, we denote by $\|\cdot\|_{2}$ and $\langle\cdot, \cdot\rangle_{2}$ the usual norm and scalar product on $L^{2}\left(\mu^{p}\right)$ for whatever value of $p$. We further define $L^{2}\left(\mu^{0}\right):=\mathbb{R}$. For $f \in L^{2}\left(\mu^{p}\right)$, we denote by $I_{p}(f)$ the multiple WienerItô integral of $f$ with respect to $\hat{\eta}$. If $p=0$, then, by convention, $I_{0}(c):=c$ for each $c \in \mathbb{R}$. We refer to Section 3 of Last (2016) for a precise definition and the following basic properties of these integrals in the general framework of a $\sigma$-finite measure space $(\mathcal{Z}, \mathscr{Z}, \mu)$. Let $p, q \geq 0$ be integers:

(1) $I_{p}(f)=I_{p}(\tilde{f})$, where $\tilde{f}$ denotes the canonical symmetrization of $f \in$ $L^{2}\left(\mu^{p}\right)$, that is, with $\mathbb{S}_{p}$ the symmetric group acting on $\{1, \ldots, p\}$ we have

$$
\tilde{f}\left(z_{1}, \ldots, z_{p}\right)=\frac{1}{p !} \sum_{\pi \in \mathbb{S}_{p}} f\left(z_{\pi(1)}, \ldots, z_{\pi(p)}\right) .
$$

(2) $I_{p}(f) \in L^{2}(\mathbb{P})$, and $\mathbb{E}\left[I_{p}(f) I_{q}(g)\right]=\delta_{p, q} p !\langle\tilde{f}, \tilde{g}\rangle_{2}$, where $\delta_{p, q}$ denotes Kronecker's delta symbol.

For $p \geq 0$, the Hilbert space consisting of all random variables $I_{p}(f), f \in$ $L^{2}\left(\mu^{p}\right)$, is called the pth Wiener chaos associated with $\eta$, and is customarily denoted by $C_{p}$. It is a crucial fact that every $F \in L^{2}(\mathbb{P})$ admits a unique representation

$$
F=\mathbb{E}[F]+\sum_{p=1}^{\infty} I_{p}\left(f_{p}\right),
$$

where $f_{p} \in L_{s}^{2}\left(\mu^{p}\right), p \geq 1$, are suitable symmetric kernel functions, and the series converges in $L^{2}(\mathbb{P})$. Identity (16) is referred to as the chaotic decomposition of the functional $F \in L^{2}(\mathbb{P})$.

From Theorem 2 in Last (2016) [which is Theorem 1.3 from the article Last and Penrose (2011)], it is known that, for all $F \in L^{2}(\mathbb{P})$ and all $p \geq 1$, the kernel $f_{p}$ in (16) is explicitly given by

$$
f_{p}\left(z_{1}, \ldots, z_{p}\right)=\frac{1}{p !} \mathbb{E}\left[D_{z_{1}, \ldots, z_{p}}^{(p)} F\right], \quad z_{1}, \ldots, z_{p} \in \mathcal{Z} .
$$


The following new lemma, which relies on (17) and whose proof is deferred to Section 6, will be essential for the proof of Theorem 1.3.

LEMMA 2.4. Let $p, q \geq 1$ be integers and let the multiple Wiener-Itô integrals $F=I_{p}(f)$ and $G=I_{q}(g)$ be in $L^{4}(\mathbb{P})$ and given by symmetric kernels $f \in L^{2}\left(\mu^{p}\right)$ and $g \in L^{2}\left(\mu^{q}\right)$, respectively.

(a) The product $F G$ has a finite chaotic decomposition of the form $F G=$ $\sum_{r=0}^{p+q} \operatorname{proj}\left\{F G \mid C_{r}\right\}=\sum_{r=0}^{p+q} I_{r}\left(h_{r}\right)$ with symmetric kernels $h_{r} \in L_{s}^{2}\left(\mu^{r}\right)$.

(b) The kernel $h_{p+q}$ in (a) is explicitly given by $h_{p+q}=f \tilde{\otimes} g$, where $f \otimes g \in$ $L^{2}\left(\mu^{p+q}\right)$ denotes the tensor product of $f$ and $g$ defined by

$$
f \otimes g\left(z_{1}, \ldots, z_{p+q}\right)=f\left(z_{1}, \ldots, z_{p}\right) g\left(z_{p+1}, \ldots, z_{p+q}\right)
$$

and $f \tilde{\otimes} g$ denotes its canonical symmetrization.

REMARK 2.5. We stress that the statement of Lemma 2.4 is not a direct consequence of the product formula for multiple Wiener-Itô integrals on the Poisson space [see, e.g., Proposition 5 in Last (2016) and the discussion therein], since such a result assumes the square-integrability of the so-called 'star contractions kernels' $f \star_{r}^{l} g$ associated with $f$ and $g$. It is easily seen that such an integrability property cannot be directly deduced from the minimal assumptions of Lemma 2.4.

2.4. $L^{2}$ theory, part 2: Malliavin operators and carré-du-champ. We now briefly discuss Malliavin operators on the Poisson space:

(i) The domain $\operatorname{dom} D$ of the Malliavin derivative operator $D$ is the set of all $F \in L^{2}(\mathbb{P})$ such that the chaotic decomposition (16) of $F$ satisfies $\sum_{p=1}^{\infty} p p !\left\|f_{p}\right\|_{2}^{2}<\infty$. For such an $F$, the random function $\mathcal{Z} \ni z \mapsto D_{z} F \in L^{2}(\mathbb{P})$ is defined via

$$
D_{z} F=\sum_{p=1}^{\infty} p I_{p-1}\left(f_{p}(z, \cdot)\right),
$$

whenever $z$ is such that the series is converging in $L^{2}(\mathbb{P})$ (this happens a.e.- $\mu$ ), and set to zero otherwise; note that $f_{p}(z, \cdot)$ is an a.e. symmetric function on $\mathcal{Z}^{p-1}$. Hence, $D F=\left(D_{z} F\right)_{z \in \mathcal{Z}}$ is indeed an element of $L^{2}(\mathbb{P} \otimes \mu)$. It is well known [see, e.g., Peccati and Thäle (2013), Lemma 3.1] that $F \in \operatorname{dom} D$ if and only if $D^{+} F \in L^{2}(\mathbb{P} \otimes \mu)$, and in this case

$$
D_{z} F=D_{z}^{+} F, \quad \mathbb{P} \otimes \mu \text {-a.e.. }
$$

(ii) The domain dom $L$ of the Ornstein-Uhlenbeck generator $L$ is the set of those $F \in L^{2}(\mathbb{P})$ whose chaotic decomposition (16) verifies $\sum_{p=1}^{\infty} p^{2} p$ ! $\left\|f_{p}\right\|_{2}^{2}<$ $\infty$ (so that $\operatorname{dom} L \subset \operatorname{dom} D$ ) and, for $F \in \operatorname{dom} L$, one defines

$$
L F=-\sum_{p=1}^{\infty} p I_{p}\left(f_{p}\right) .
$$


By definition, $\mathbb{E}[L F]=0$; also, from (20) it is easy to see that $L$ is symmetric in the sense that

$$
\mathbb{E}[(L F) G]=\mathbb{E}[F(L G)]
$$

for all $F, G \in \operatorname{dom} L$. Note that, from (20), it is immediate that the spectrum of $-L$ is given by the nonnegative integers and that $F \in \operatorname{dom} L$ is an eigenfunction of $-L$ with corresponding eigenvalue $p$ if and only if $F=I_{p}\left(f_{p}\right)$ for some $f_{p} \in L_{s}^{2}\left(\mu^{p}\right)$, that is,

$$
C_{p}=\operatorname{Ker}(L+p I) .
$$

For $F \in L^{2}(\mathbb{P})$ given by (16) and $p \in \mathbb{N}_{0}$, we write

$$
\operatorname{proj}\left\{F \mid C_{p}\right\}=I_{p}\left(f_{p}\right)
$$

for the projection of $F$ onto $C_{p}$, with $f_{0}:=\mathbb{E}[F]$. The following identity, which corresponds to formula (65) in Last (2016), will play an important role in the sequel: if $F \in \operatorname{dom} L$ is such that $D^{+} F \in L^{1}(\mathbb{P} \otimes \mu)$, then

$$
L F=\int_{\mathcal{Z}}\left(D_{z}^{+} F\right) \mu(d z)-\int_{\mathcal{Z}}\left(D_{z}^{-} F\right) \eta(d z) .
$$

(iii) In order to deal with bounds in the Kolmogorov distance, we will also exploit the properties of the Skohorod integral operator $\delta$ associated with $\eta$, which is characterised by the following duality relation:

$$
\mathbb{E}[G \delta(u)]=\mathbb{E}\left[\langle D G, u\rangle_{L^{2}(\mu)}\right] \quad \text { for all } G \in \operatorname{dom} D, u \in \operatorname{dom} \delta,
$$

where $\operatorname{dom} \delta$ stands for its domain [see Last (2016), pages 14-15]. Recall that the operator $\delta$ satisfies the classical identity

$$
L=-\delta D \text {, }
$$

that has to be understood in the following sense: $F \in \operatorname{dom} L$ if and only if $F \in$ $\operatorname{dom} D$ and $D F \in \operatorname{dom} \delta$, and in this case $\delta D F=-L F$. Also, if $u(\eta, \cdot) \in L^{1}(\mathbb{P} \otimes$ $\mu) \cap \operatorname{dom} \delta$, then

$$
\delta(u)=\int_{\mathcal{Z}} u\left(\eta-\delta_{z}, z\right) \eta(d z)-\int_{\mathcal{Z}} u(\eta, z) \mu(d z) \quad \text { a.s.- } \mathbb{P} ;
$$

see Last (2016), Theorem 6, for a proof of this fact.

(iv) As it is customary in the theory of Markov generators [see, e.g., Bakry, Gentil and Ledoux (2014)], for suitable random variables $F, G \in \operatorname{dom} L$ such that $F G \in \operatorname{dom} L$, we introduce the carré-du-champ operator $\Gamma$ associated with $L$ by

$$
\Gamma(F, G):=\frac{1}{2}(L(F G)-F L G-G L F) .
$$

The symmetry of $L$ implies immediately the crucial integration by parts formula

$$
\mathbb{E}[(L F) G]=\mathbb{E}[F(L G)]=-\mathbb{E}[\Gamma(F, G)] .
$$

The connection between (26) and (15) will be clarified in the discussion to follow. 
(v) The domain dom $L^{-1}$ of the pseudo-inverse $L^{-1}$ of $L$ is the class of mean zero elements $F$ of $L^{2}(\mathbb{P})$. If $F=\sum_{p=1}^{\infty} I_{p}\left(f_{p}\right)$ is the chaotic decomposition of $F$, then $L^{-1} \mathrm{~F}$ is given by

$$
L^{-1} F=-\sum_{p=1}^{\infty} \frac{1}{p} I_{p}(f) .
$$

Note that these definitions imply that $L^{-1} F \in \operatorname{dom} L$ (and therefore $L^{-1} F \in$ $\operatorname{dom} D$ ), for every $F \in \operatorname{dom} L^{-1}$, and moreover

$$
\begin{aligned}
& L L^{-1} F=F \quad \text { for all } F \in \operatorname{dom} L^{-1} \text { and } \\
& L^{-1} L F=F-\mathbb{E}[F] \quad \text { for all } F \in \operatorname{dom} L .
\end{aligned}
$$

Using the first of these identities as well as (26) we obtain that, for $F, G$ such that $G, G L^{-1}(F-\mathbb{E}(F)) \in \operatorname{dom} L$,

$$
\begin{aligned}
\operatorname{Cov}(F, G) & =\mathbb{E}[G(F-\mathbb{E}[F])]=\mathbb{E}\left[G \cdot L L^{-1}(F-\mathbb{E}[F])\right] \\
& =-\mathbb{E}\left[\Gamma\left(G, L^{-1}(F-\mathbb{E}[F])\right] .\right.
\end{aligned}
$$

In particular, if $F=I_{q}(f)$ is a multiple integral of order $q \geq 1$ such that $F^{2} \in$ $\operatorname{dom} L$, then $\mathbb{E}[F]=0, L^{-1} F=-q^{-1} F$ and

$$
\operatorname{Var}(F)=\frac{1}{q} \mathbb{E}[\Gamma(F, F)] .
$$

Note that Lemma 2.4 immediately implies that $F^{2}=I_{q}(f)^{2} \in \operatorname{dom} L$ if and only if $F \in L^{4}(\mathbb{P})$. On the other hand, if $G \in \operatorname{dom} D$ and $G D^{+}\left(L^{-1} F\right), D^{+}\left(L^{-1} F\right) \in$ $L^{1}(\mathbb{P} \otimes \mu)$, then combining (in order) (21), (15) and (19) yields

$$
\operatorname{Cov}(F, G)=\mathbb{E}\left[G \cdot L L^{-1}(F-\mathbb{E}[F])\right]=-\mathbb{E}\left[\Gamma_{0}\left(G, L^{-1}(F-\mathbb{E}[F])\right)\right]
$$

2.5. Combining $L^{1}$ and $L^{2}$ techniques. The following result provides an explicit representation of the carré-du-champ operator $\Gamma$ in terms of $\Gamma_{0}$, as introduced in (14). Although such a characterisation follows quite straightforwardly from the (classical) results and definitions provided above, we were not able to locate it in the existing literature [at least not at our level of generality; see, e.g., Bouleau and Denis (2015), Proposition 4.7, for a similar statement in a more restrictive setting] and we will therefore provide a full proof. It is one of the staples of our approach.

Proposition 2.6. For all $F, G \in \operatorname{dom} L$ such that $F G \in \operatorname{dom} L$ and

$$
D F, D G, F D G, G D F \in L^{1}(\mathbb{P} \otimes \mu),
$$

we have that $D F=D^{+} F, D G=D^{+} G$, in such a way that $D F D G=$ $D^{+} F D^{+} G \in L^{1}(\mathbb{P} \otimes \mu)$, and

$$
\Gamma(F, G)=\Gamma_{0}(F, G),
$$

where $\Gamma_{0}$ is defined in (14). 
In order to prove Proposition 2.6, we state the following lemma which will be exploited in several occasions.

Lemma 2.7. (a) For $F \in \mathcal{L}^{0}(\Omega)$ and $z \in \mathcal{Z}$, we have the identities:

$$
\begin{aligned}
& D_{z}^{+} F^{2}=\left(D_{z}^{+} F\right)^{2}+2 F D_{z}^{+} F, \\
& D_{z}^{+} F^{3}=\left(D_{z}^{+} F\right)^{3}+3 F^{2} D_{z}^{+} F+3 F\left(D_{z}^{+} F\right)^{2}, \\
& D_{z}^{-} F^{2}=-\left(D_{z}^{-} F\right)^{2}+2 F D_{z}^{-} F, \\
& D_{z}^{-} F^{3}=\left(D_{z}^{-} F\right)^{3}+3 F^{2} D_{z}^{-} F-3 F\left(D_{z}^{-} F\right)^{2} .
\end{aligned}
$$

(b) Let $\psi \in C^{1}(\mathbb{R})$ be such that $\psi^{\prime}$ is Lipschitz with minimum Lipschitz-constant $\left\|\psi^{\prime \prime}\right\|_{\infty}$. Then, for $F \in \mathcal{L}^{0}(\Omega)$ and $z \in \mathcal{Z}$, there are random quantities $R_{\psi}^{+}(F, z)$ and $R_{\psi}^{-}(F, z)$ such that

$$
\left|R_{\psi}^{+}(F, z)\right| \leq \frac{\left\|\psi^{\prime \prime}\right\|_{\infty}}{2}, \quad\left|R_{\psi}^{-}(F, z)\right| \leq \frac{\left\|\psi^{\prime \prime}\right\|_{\infty}}{2}
$$

and

$$
\begin{aligned}
& D_{z}^{+} \psi(F)=\psi^{\prime}(F) D_{z}^{+} F+R_{\psi}^{+}(F, z)\left(D_{z}^{+} F\right)^{2} \text { and } \\
& D_{z}^{-} \psi(F)=\psi^{\prime}(F) D_{z}^{-} F+R_{\psi}^{-}(F, z)\left(D_{z}^{-} F\right)^{2} .
\end{aligned}
$$

PROOF. The proof of this result is deferred to Section 6.

REMARK 2.8. Note that, by virtue of (31) and polarization, for $F, G \in \mathcal{L}^{0}(\Omega)$ and $z \in \mathcal{Z}$ we also deduce the product rules:

$$
\begin{aligned}
& D_{z}^{+}(F G)=G D_{z}^{+} F+F D_{z}^{+} G+\left(D_{z}^{+} F\right)\left(D_{z}^{+} G\right), \\
& D_{z}^{-}(F G)=G D_{z}^{-} F+F D_{z}^{-} G-\left(D_{z}^{-} F\right)\left(D_{z}^{-} G\right) .
\end{aligned}
$$

If, furthermore, $F, G, F G \in \operatorname{dom} D$, then, from (19) we conclude that

$$
D_{z}(F G)=G D_{z} F+F D_{z} G+\left(D_{z} F\right)\left(D_{z} G\right), \quad z \in \mathcal{Z},
$$

for the Malliavin derivative $D$. Relations (35)-(36) combined with (30) imply that $\Gamma$ is not a derivation, and confirm the well-known fact that $L$ is not a diffusion operator [see, e.g., Bakry, Gentil and Ledoux (2014), Definition 1.11.1, for definitions].

Proof of Proposition 2.6. We need only prove (30), as the rest of the assertions in the statement follows from elementary considerations. Since our assumptions imply that $D(F G) \in L^{1}(\mathbb{P} \otimes \mu)$, we can apply (21) in order to deduce 
that

$$
\begin{aligned}
2 \Gamma(F, G)= & L F G-G L F-F L G \\
= & \int_{\mathcal{Z}} D_{z}^{+}(F G) \mu(d z)-\int_{\mathcal{Z}} D_{z}^{-}(F G) \eta(d z) \\
& -G \int_{\mathcal{Z}} D_{z}^{+} F \mu(d z)+G \int_{\mathcal{Z}} D_{z}^{-} F \eta(d z) \\
& -F \int_{\mathcal{Z}} D_{z}^{+} G \mu(d z)+F \int_{\mathcal{Z}} D_{z}^{-} G \eta(d z) .
\end{aligned}
$$

Using (35) and (36) yields immediately the desired formula.

3. Identities and estimates for multiple integrals. We will now prove several important relations involving multiple stochastic integrals of a fixed order $q \geq 1$. They constitute the backbone of the forthcoming proof of Theorem 1.3.

LEMMA 3.1. Let $q \geq 1$, and consider a random variable $F$ such that $F=$ $I_{q}(f) \in C_{q}=\operatorname{Ker}(L+q I)$ and $\mathbb{E}\left[F^{4}\right]<\infty$. Then $F, F^{2} \in \operatorname{dom} L$, and

$$
\begin{aligned}
\operatorname{Var}\left(q^{-1} \Gamma(F, F)\right) & =\sum_{p=1}^{2 q-1}\left(1-\frac{p}{2 q}\right)^{2} \operatorname{Var}\left(\operatorname{proj}\left\{F^{2} \mid C_{p}\right\}\right) \\
& \leq \frac{(2 q-1)^{2}}{4 q^{2}}\left(\mathbb{E}\left[F^{4}\right]-3 \mathbb{E}\left[F^{2}\right]^{2}\right)
\end{aligned}
$$

Moreover, one has also that

$$
\begin{gathered}
\frac{1}{q^{2}} \mathbb{E}\left[\Gamma(F, F)^{2}\right] \leq \mathbb{E}\left[F^{4}\right], \\
\frac{1}{q} \mathbb{E}\left[F^{2} \Gamma(F, F)\right] \leq \mathbb{E}\left[F^{4}\right]
\end{gathered}
$$

PROOF. From Lemma 2.4, we know that $F^{2}=I_{q}(f)^{2}$ has a chaos decomposition of the form

$$
F^{2}=\sum_{p=0}^{2 q} \operatorname{proj}\left\{F^{2} \mid C_{p}\right\}=\mathbb{E}\left[F^{2}\right]+\sum_{p=1}^{2 q-1} \operatorname{proj}\left\{F^{2} \mid C_{p}\right\}+I_{2 q}\left(g_{2 q}\right)
$$

with $g_{2 q}=f \tilde{\otimes} f$, thus ensuring that $F^{2}$ is in the domain of $L$. By homogeneity, without loss of generality we can assume for the rest of the proof that $\mathbb{E}\left[F^{2}\right]=1$. 
As $L F=-q F$, by the definitions of $\Gamma$ and $L$ we have

$$
\begin{aligned}
2 \Gamma(F, F) & =L F^{2}-2 F L F \\
& =\sum_{p=1}^{2 q}-p \operatorname{proj}\left\{F^{2} \mid C_{p}\right\}+2 q \sum_{p=0}^{2 q} \operatorname{proj}\left\{F^{2} \mid C_{p}\right\} \\
& =\sum_{p=0}^{2 q}(2 q-p) \operatorname{proj}\left\{F^{2} \mid C_{p}\right\} .
\end{aligned}
$$

By orthogonality, one has that

$$
\begin{aligned}
\operatorname{Var}\left(q^{-1} \Gamma(F, F)\right) & =\frac{1}{4 q^{2}} \sum_{p=1}^{2 q}(2 q-p)^{2} \operatorname{Var}\left(\operatorname{proj}\left\{F^{2} \mid C_{p}\right\}\right) \\
& =\frac{1}{4 q^{2}} \sum_{p=1}^{2 q-1}(2 q-p)^{2} \operatorname{Var}\left(\operatorname{proj}\left\{F^{2} \mid C_{p}\right\}\right),
\end{aligned}
$$

proving the first equality in (38). For the inequality, first note that from (41) and the isometry property of multiple integrals we have

$$
\begin{aligned}
\mathbb{E}\left[F^{4}\right]-1 & =\operatorname{Var}\left(F^{2}\right)=\sum_{p=1}^{2 q} \operatorname{Var}\left(\operatorname{proj}\left\{F^{2} \mid C_{p}\right\}\right) \\
& =\sum_{p=1}^{2 q-1} \operatorname{Var}\left(\operatorname{proj}\left\{F^{2} \mid C_{p}\right\}\right)+(2 q) !\|f \tilde{\otimes} f\|_{2}^{2} .
\end{aligned}
$$

Now, identity (5.2.12) in the book Nourdin and Peccati (2012) yields that

$$
(2 q !)\|f \tilde{\otimes} f\|_{2}^{2}=2(q !)^{2}\|f\|_{2}^{4}+D_{q},
$$

where $D_{q} \geq 0$ is a finite nonnegative quantity that can be expressed in terms of the contraction kernels associated with $F$, and whose explicit form is immaterial for the present proof. Also,

$$
2(q !)^{2}\|f\|_{2}^{4}=2\left(\mathbb{E}\left[F^{2}\right]\right)^{2}=2,
$$

and we deduce from (43) and (44) that

$$
\begin{aligned}
\frac{(2 q-1)^{2}}{4 q^{2}}\left(\mathbb{E}\left[F^{4}\right]-3\right) & =\frac{(2 q-1)^{2}}{4 q^{2}} \sum_{p=1}^{2 q-1} \operatorname{Var}\left(\operatorname{proj}\left\{F^{2} \mid C_{p}\right\}\right)+\frac{(2 q-1)^{2}}{4 q^{2}} D_{q} \\
& \geq \frac{(2 q-1)^{2}}{4 q^{2}} \sum_{p=1}^{2 q-1} \operatorname{Var}\left(\operatorname{proj}\left\{F^{2} \mid C_{p}\right\}\right)
\end{aligned}
$$




$$
\begin{aligned}
& \geq \frac{1}{4 q^{2}} \sum_{p=1}^{2 q-1}(2 q-p)^{2} \operatorname{Var}\left(\operatorname{proj}\left\{F^{2} \mid C_{p}\right\}\right) \\
& =\operatorname{Var}\left(q^{-1} \Gamma(F, F)\right),
\end{aligned}
$$

which is exactly the second estimate in (38). Relations (39) and (40) are immediate consequences of (41) and (42).

The following result will allow us to effectively control residual quantities arising from the application of Stein's method on the Poisson space.

LEMMA 3.2. Let $q \geq 1$ be an integer and let $F \in L^{4}(\mathbb{P})$ be an element of the $q$ th Wiener chaos $C_{q}$, such that $F$ verifies Assumption A. Then

$$
\begin{aligned}
\frac{1}{2 q} \int_{\mathcal{Z}} \mathbb{E}\left[\left|D_{z}^{+} F\right|^{4}\right] \mu(d z) & =\frac{3}{q} \mathbb{E}\left[F^{2} \Gamma(F, F)\right]-\mathbb{E}\left[F^{4}\right] \\
& \leq \frac{4 q-3}{2 q}\left(\mathbb{E}\left[F^{4}\right]-3 \mathbb{E}\left[F^{2}\right]^{2}\right) .
\end{aligned}
$$

PROOF. Again by homogeneity, we can assume without loss of generality that $F$ has unit variance. Observe that $F \in \operatorname{dom} D$ and, therefore, $D F=D^{+} F$ (up to a $\mathbb{P} \otimes \mu$-negligible set), and also, by virtue of Proposition 2.6, one has that $\Gamma(F, F)=\Gamma_{0}(F, F)$, a.s.-P. It follows that

$$
\begin{aligned}
\mathbb{E}\left[F^{2} \int_{\mathcal{Z}}\left(D_{z}^{+} F\right)^{2} \mu(d z)\right] & \leq 2 \mathbb{E}\left[F^{2} \Gamma_{0}(F, F)\right]=2 \mathbb{E}\left[F^{2} \Gamma(F, F)\right] \\
& \leq 2 q \mathbb{E}\left[F^{4}\right]<\infty,
\end{aligned}
$$

where we have used (40) as well as the fact that the integral of a non-negative function with respect to the noncompensated Poisson measure $\eta$ is nonnegative. Moreover, by Cauchy-Schwarz,

$$
\begin{aligned}
\mathbb{E}\left[|F| \int_{\mathcal{Z}}\left|D_{z}^{+} F\right|^{3} \mu(d z)\right] & \leq \mathbb{E}\left[F^{2} \int_{\mathcal{Z}}\left(D_{z}^{+} F\right)^{2} \mu(d z)\right]^{1 / 2} \mathbb{E}\left[\int_{\mathcal{Z}}\left(D_{z}^{+} F\right)^{4} \mu(d z)\right]^{1 / 2} \\
& <\infty,
\end{aligned}
$$

so that $F^{2}\left(D^{+} F\right)^{2}, F\left(D^{+} F\right)^{3} \in L^{1}(\mathbb{P} \otimes \mu)$. Since $L F=-q F$ and $D F \in L^{1}(\mathbb{P} \otimes$ $\mu)$, one infers from (21) that

$$
F=-\frac{1}{q}\left(\int_{\mathcal{Z}}\left(D_{z}^{+} F\right) \mu(d z)-\int_{\mathcal{Z}}\left(D_{z}^{-} F\right) \eta(d z)\right) .
$$

Since the above discussion also implies that $F^{3} D^{+} F, D^{+}\left(F^{3}\right) D^{+} F \in L^{1}(\mathbb{P} \otimes \mu)$ [via (32)], we can now exploit the integration by parts relation stated in Lemma 2.3 to deduce that

$$
\mathbb{E}\left[F^{4}\right]=-\frac{1}{q} \mathbb{E}\left[F^{3}\left(\int_{\mathcal{Z}}\left(D_{z}^{+} F\right) \mu(d z)-\int_{\mathcal{Z}}\left(D_{z}^{-} F\right) \eta(d z)\right)\right]=\frac{1}{q} \mathbb{E}\left[\Gamma_{0}\left(F, F^{3}\right)\right] .
$$


Now, using (32) and (34) we obtain

$$
\begin{aligned}
\Gamma_{0}\left(F, F^{3}\right)= & \frac{1}{2}\left(\int_{\mathcal{Z}} D_{z}^{+} F\left(\left(D_{z}^{+} F\right)^{3}+3 F^{2} D_{z}^{+} F+3 F\left(D_{z}^{+} F\right)^{2}\right) \mu(d z)\right. \\
& \left.+\int_{\mathcal{Z}} D_{z}^{-} F\left(\left(D_{z}^{-} F\right)^{3}+3 F^{2} D_{z}^{-} F-3 F\left(D_{z}^{-} F\right)^{2}\right) \eta(d z)\right) \\
= & \frac{1}{2}\left(\int_{\mathcal{Z}}\left(\left(D_{z}^{+} F\right)^{4}+3 F^{2}\left(D_{z}^{+} F\right)^{2}+3 F\left(D_{z}^{+} F\right)^{3}\right) \mu(d z)\right. \\
& \left.+\int_{\mathcal{Z}}\left(\left(D_{z}^{-} F\right)^{4}+3 F^{2}\left(D_{z}^{-} F\right)^{2}-3 F\left(D_{z}^{-} F\right)^{3}\right) \eta(d z)\right),
\end{aligned}
$$

and we also have

$$
\begin{aligned}
3 F^{2} \Gamma_{0}(F, F) & =3 F^{2} \Gamma(F, F) \\
& =\frac{1}{2}\left(\int_{\mathcal{Z}} 3 F^{2}\left(D_{z}^{+} F\right)^{2} \mu(d z)+\int_{\mathcal{Z}} 3 F^{2}\left(D_{z}^{-} F\right)^{2} \eta(d z)\right) .
\end{aligned}
$$

Hence, using the Mecke formula (13) (as well as the content of Remark 2.2) in the case

$$
V(z)=-\left(\mathfrak{f}\left(\eta+\delta_{z}\right)-\mathfrak{f}(\eta)\right)^{4}-3 \mathfrak{f}(\eta)\left(\mathfrak{f}\left(\eta+\delta_{z}\right)-\mathfrak{f}(\eta)\right)^{3},
$$

where $\mathfrak{f}$ is some representative of $F$, we can conclude that

$$
\begin{aligned}
\frac{3}{q} \mathbb{E}[ & \left.F^{2} \Gamma(F, F)\right]-\mathbb{E}\left[F^{4}\right] \\
= & \frac{1}{2 q} \mathbb{E}\left[\int_{\mathcal{Z}}\left(-\left(D_{z}^{+} F\right)^{4}-3 F\left(D_{z}^{+} F\right)^{3}\right) \mu(d z)\right. \\
& \left.+\int_{\mathcal{Z}}\left(-\left(D_{z}^{-} F\right)^{4}+3 F\left(D_{z}^{-} F\right)^{3}\right) \eta(d z)\right] \\
= & \frac{1}{2 q} \mathbb{E}\left[-2 \int_{\mathcal{Z}}\left(D_{z}^{+} F\right)^{4} \mu(d z)+3 \int_{\mathcal{Z}}\left(D_{z}^{+} F\right)^{3}\left(\mathfrak{f}\left(\eta+\delta_{z}\right)-\mathfrak{f}(\eta)\right) \mu(d z)\right] \\
= & \frac{1}{2 q} \mathbb{E}\left[\int_{\mathcal{Z}}\left(D_{z}^{+} F\right)^{4} \mu(d z)\right] .
\end{aligned}
$$

Finally, using relations (41) and (42) from the proof of Lemma 3.1, we obtain

$$
\begin{array}{rl}
\frac{1}{q} \int_{\mathcal{Z}} & \mathbb{E}\left[\left|D_{z}^{+} F\right|^{4}\right] \mu(d z) \\
& =2\left(\frac{3}{q} \mathbb{E}\left[F^{2} \Gamma(F, F)\right]-\mathbb{E}\left[F^{4}\right]\right) \\
& =2\left(\frac{3}{q} q\left(\mathbb{E}\left[F^{2}\right]\right)^{2}-\mathbb{E}\left[F^{4}\right]+\frac{3}{2 q} \sum_{p=1}^{2 q-1}(2 q-p) \operatorname{Var}\left(\operatorname{proj}\left\{F^{2} \mid C_{p}\right\}\right)\right)
\end{array}
$$




$$
\begin{aligned}
& \leq 2\left(3-\mathbb{E}\left[F^{4}\right]\right)+\frac{3(2 q-1)}{q} \sum_{p=1}^{2 q-1} \operatorname{Var}\left(\operatorname{proj}\left\{F^{2} \mid C_{p}\right\}\right) \\
& \leq 2\left(3-\mathbb{E}\left[F^{4}\right]\right)+\frac{3(2 q-1)}{q}\left(\mathbb{E}\left[F^{4}\right]-3\right) \\
& =\frac{4 q-3}{q}\left(\mathbb{E}\left[F^{4}\right]-3\right),
\end{aligned}
$$

where the last inequality is again a consequence of (43) and (44).

We eventually prove an estimate that will be crucial in order to deal with bounds in the Kolmogorov distance.

LEMMA 3.3. For some fixed $q \geq 1$, let $F \in \operatorname{Ker}(L+q I)$ satisfy both Assumption $\mathrm{A}$ and Assumption $\mathrm{A}^{\text {loc }}$. Then

$$
0 \leq \frac{1}{q} \sup _{x \in \mathbb{R}} \mathbb{E}\left[\int_{\mathcal{Z}}\left(D_{z}^{+} \mathbf{1}_{\{F>x\}}\left|D_{z}^{+} F\right| D_{z}^{+} F \mu(d z)\right] \leq 10 \sqrt{\mathbb{E}\left[F^{4}\right]-3 \mathbb{E}\left[F^{2}\right]^{2}}\right.
$$

Proof. One checks immediately that $D_{z}^{+} \mathbf{1}_{\{F>x\}} D_{z}^{+} F \geq 0$, so that we need only prove the second inequality in the statement; also, without loss of generality and by homogeneity, we can once again assume that $F$ has unit variance. According to (18)-(19), we can choose a version of $D^{+} F$ such that, for $\mu$-almost every $z \in \mathcal{Z}$, the random variable $D_{z}^{+} F=D_{z} F$ is an element of the $(q-1)$ th Wiener chaos $C_{q-1}$. Applying Lemma 3.1 and Lemma 3.2 to every $D_{z} F$ such that $z$ lies outside the exceptional set, one therefore infers that

$$
\begin{aligned}
A & :=\int_{\mathcal{Z}} \mathbb{E}\left[\int_{\mathcal{Z}}\left(D_{z_{2}} D_{z_{1}} F\right)^{4} \mu\left(d z_{2}\right)\right] \mu\left(d z_{1}\right) \\
& =\int_{\mathcal{Z}} \mathbb{E}\left[\int_{\mathcal{Z}}\left(D_{z_{2}}^{+}\left(D_{z_{1}}^{+} F\right)\right)^{4} \mu\left(d z_{2}\right)\right] \mu\left(d z_{1}\right) \\
& \leq 4(q-1) \mathbb{E}\left[\int_{\mathcal{Z}}\left(D_{z}^{+} F\right)^{4} \mu(d z)\right] \leq 16 q(q-1)\left(\mathbb{E}\left[F^{4}\right]-3\right),
\end{aligned}
$$

where we have applied twice (first to $D_{z_{1}}^{+} F$ and then to $F$ itself) the following inequality which is immediate from Lemma 3.2:

$$
C:=\int_{\mathcal{Z}} \mathbb{E}\left[\left|D_{z}^{+} F\right|^{4}\right] \mu(d z) \leq 4 q\left(\mathbb{E}\left[F^{4}\right]-3\right)
$$


Moreover, using the definition (14) of $\Gamma_{0}$, as well as (40) and again (48) we obtain

$$
\begin{aligned}
B & :=\int_{\mathcal{Z}} \mathbb{E}\left[\left(D_{z_{1}} F\right)^{2} \int_{\mathcal{Z}}\left(D_{z_{2}} D_{z_{1}} F\right)^{2} \mu\left(d z_{2}\right)\right] \mu\left(d z_{1}\right) \\
& =\int_{\mathcal{Z}} \mathbb{E}\left[\left(D_{z_{1}}^{+} F\right)^{2} \int_{\mathcal{Z}}\left(D_{z_{2}}^{+} D_{z_{1}}^{+} F\right)^{2} \mu\left(d z_{2}\right)\right] \mu\left(d z_{1}\right) \\
& \leq 2(q-1) \int_{\mathcal{Z}} \mathbb{E}\left[\left(D_{z_{1}}^{+} F\right)^{2} \Gamma_{0}\left(D_{z_{1}}^{+} F, D_{z_{1}}^{+} F\right)\right] \mu\left(d z_{1}\right) \\
& =2(q-1) \int_{\mathcal{Z}} \mathbb{E}\left[\left(D_{z_{1}}^{+} F\right)^{2} \Gamma\left(D_{z_{1}}^{+} F, D_{z_{1}}^{+} F\right)\right] \mu\left(d z_{1}\right) \\
& \leq 2(q-1) \mathbb{E}\left[\int_{\mathcal{Z}}\left(D_{z_{1}}^{+} F\right)^{4} \mu\left(d z_{1}\right)\right] \leq 8 q(q-1)\left(\mathbb{E}\left[F^{4}\right]-3\right) .
\end{aligned}
$$

Now write $\Phi(a):=a|a|, a \in \mathbb{R}$. In view of the inequality [proved, e.g., in Peccati and Thäle (2013), Section 4.2]

$$
\left[D_{z_{2}}^{+} \Phi\left(D_{z_{1}}^{+} F\right)\right]^{2} \leq 8\left(D_{z_{1}}^{+} F\right)^{2}\left(D_{z_{2}}^{+} D_{z_{1}}^{+} F\right)^{2}+2\left(D_{z_{2}}^{+} D_{z_{1}}^{+} F\right)^{4},
$$

valid $\mu^{2}$-almost everywhere, we deduce immediately that the process $z \mapsto v(z):=$ $\Phi\left(D_{z}^{+} F\right)$ is such that $v(z) \in \operatorname{dom} D$ for $\mu$-almost every $z$, and $v \in \operatorname{dom} \delta$-this last fact being a consequence of the classical criterion stated in Last (2016) and of the estimates (47)-(49), Theorem 5, together with the fact that $\mathbb{E}\left[F^{4}\right]<\infty$ by assumption. Also, in view of the fact that $v \in L^{1}(\mathbb{P} \otimes \mu)$ by assumption, equation (24) yields that

$$
\delta(v)=\int_{\mathcal{Z}} \Phi\left(D_{z}^{-} F\right) \eta(d z)-\int_{\mathcal{Z}} \Phi\left(D_{z}^{+} F\right) \mu(d z) .
$$

We now fix $x \in \mathbb{R}$. Relation (13) applied to the mapping

$$
V(z)=\mathbf{1}_{\left\{\mathfrak{f}\left(\eta+\delta_{z}\right)>x\right\}} \Phi\left(\mathfrak{f}\left(\eta+\delta_{z}\right)-\mathfrak{f}(\eta)\right),
$$

where $\mathfrak{f}$ is a representative of $F$, yields that

$$
\begin{aligned}
& \frac{1}{q} \mathbb{E}\left[\int_{\mathcal{Z}} D_{z}^{+} \mathbf{1}_{\{F>x\}}\left|D_{z}^{+} F\right| D_{z}^{+} F \mu(d z)\right] \\
& =\frac{1}{q} \mathbb{E}\left[\mathbf{1}_{\{F>x\}}\left(\int_{\mathcal{Z}} \Phi\left(D_{z}^{-} F\right) \eta(d z)-\int_{\mathcal{Z}} \Phi\left(D_{z}^{+} F\right) \mu(d z)\right)\right] \\
& =\frac{1}{q} \mathbb{E}\left[\mathbf{1}_{\{F>x\}} \delta(v)\right] \leq \frac{1}{q} \mathbb{E}\left[\delta(v)^{2}\right]^{1 / 2} .
\end{aligned}
$$

To conclude, we use Last (2016), formula (56), as well as (50) to deduce that

$$
\begin{aligned}
\mathbb{E}\left[\delta(v)^{2}\right] & \leq \mathbb{E}\left[\int_{\mathcal{Z}} v(z)^{2} \mu(d z)\right]+\mathbb{E}\left[\int_{\mathcal{Z}} \int_{\mathcal{Z}}\left(D_{y}^{+} v(z)\right)^{2} \mu(d z) \mu(d y)\right] \\
& \leq C+8 B+2 A \leq(4 q+64 q(q-1)+32 q(q-1))\left(\mathbb{E}\left[F^{4}\right]-3\right) \\
& \leq 100 q^{2}\left(\mathbb{E}\left[F^{4}\right]-3\right),
\end{aligned}
$$


which in turn implies that

$$
\frac{1}{q} \mathbb{E}\left[\delta(v)^{2}\right]^{1 / 2} \leq 10 \sqrt{\mathbb{E}\left[F^{4}\right]-3}
$$

where $A, B, C$ have been defined above, and where we have used the estimates (47)-(48).

4. Proof of Theorem 1.3. In order to prove Theorem 1.3, we have to establish new abstract bounds on the normal approximation of functionals on the Poisson space in the Wasserstein and Kolmogorov distances, respectively. Recall the definition of $\Gamma_{0}$ given in (14).

Proposition 4.1. Let $F \in \operatorname{dom} D$ be such that $\mathbb{E}[F]=0$ and let $N \sim$ $\mathscr{N}(0,1)$ be a standard normal random variable. Assume that

$$
D^{+}\left(L^{-1} F\right), F D^{+}\left(L^{-1} F\right) \in L^{1}(\mathbb{P} \otimes \mu) .
$$

Then we have the bounds

$$
\begin{aligned}
d_{1}(F, N) \leq & \sqrt{\frac{2}{\pi}} \mathbb{E}\left|1-\Gamma_{0}\left(F,-L^{-1} F\right)\right|+\int_{\mathcal{Z}} \mathbb{E}\left[\left|D_{z}^{+} F\right|^{2}\left|D_{z}^{+} L^{-1} F\right|\right] \mu(d z) \\
\leq & \sqrt{\frac{2}{\pi}}\left|1-\mathbb{E}\left[F^{2}\right]\right|+\sqrt{\frac{2}{\pi}} \sqrt{\operatorname{Var}\left(\Gamma_{0}\left(F,-L^{-1} F\right)\right)} \\
& +\int_{\mathcal{Z}} \mathbb{E}\left[\left|D_{z}^{+} F\right|^{2}\left|D_{z}^{+} L^{-1} F\right|\right] \mu(d z) .
\end{aligned}
$$

If, furthermore, $F=I_{q}(f)$ for some $q \geq 1$ and some square-integrable, symmetric kernel $f$ on $\mathcal{Z}^{q}$ and $\mathbb{E}\left[F^{2}\right]=q !\|f\|_{2}^{2}=1$, then $-L^{-1} F=q^{-1} F$,

$$
\begin{aligned}
\mathbb{E}\left[\Gamma_{0}\left(F,-L^{-1} F\right)\right] & =q^{-1} \mathbb{E}\left[\Gamma_{0}(F, F)\right]=1 \quad \text { and } \\
\int_{\mathcal{Z}} \mathbb{E}\left[\left|D_{z}^{+} F\right|^{2}\left|D_{z}^{+} L^{-1} F\right|\right] \mu(d z) & =q^{-1} \int_{\mathcal{Z}} \mathbb{E}\left[\left|D_{z}^{+} F\right|^{3}\right] \mu(d z) \\
& \leq\left(q^{-1} \int_{\mathcal{Z}} \mathbb{E}\left[\left|D_{z}^{+} F\right|^{4}\right] \mu(d z)\right)^{1 / 2}
\end{aligned}
$$

so that the previous estimate (53) gives

$$
d_{1}(F, N) \leq \sqrt{\frac{2}{\pi}} \sqrt{\operatorname{Var}\left(q^{-1} \Gamma_{0}(F, F)\right)}+\frac{1}{\sqrt{q}}\left(\int_{\mathcal{Z}} \mathbb{E}\left[\left|D_{z}^{+} F\right|^{4}\right] \mu(d z)\right)^{1 / 2}
$$

REMARK 4.2. Under the assumptions of Proposition 4.1, we have that $F, L^{-1} F \in \operatorname{dom} D$, in such a way that $\Gamma_{0}\left(F,-L^{-1} F\right)$ is an element of $L^{1}(\mathbb{P})$. It follows that the variance $\operatorname{Var}\left(\Gamma_{0}\left(F,-L^{-1} F\right)\right)$ is always well defined, albeit possibly infinite. 
ProOF OF Proposition 4.1. We apply Stein's method for normal approximation. Define the class $\mathscr{F}_{1}$ of all continuously differentiable functions $\psi$ on $\mathbb{R}$ such that both $\psi$ and $\psi^{\prime}$ are Lipschitz-continuous with minimal Lipschitz constants

$$
\left\|\psi^{\prime}\right\|_{\infty} \leq \sqrt{\frac{2}{\pi}} \text { and }\left\|\psi^{\prime \prime}\right\|_{\infty} \leq 2 .
$$

Then, it is well known [see, e.g., Theorem 3 of Bourguin and Peccati (2016), and the references therein] that

$$
d_{1}(F, N) \leq \sup _{\psi \in \mathscr{F}_{1}}\left|\mathbb{E}\left[\psi^{\prime}(F)-F \psi(F)\right]\right|
$$

Let us thus fix $\psi \in \mathscr{F}_{1}$. The Lipschitz property of $\psi$ implies that $\psi(F) \in \operatorname{dom} D$, whereas the trivial estimate

$$
\left|\psi(F) D^{+}\left(L^{-1} F\right)\right| \leq(|\psi(0)|+\sqrt{2 / \pi}|F|) \times\left|D^{+}\left(L^{-1} F\right)\right|
$$

implies that $\psi(F) D^{+}\left(L^{-1} F\right) \in L^{1}(\mathbb{P} \otimes \mu)$. Using that $\mathbb{E}[F]=0$, we therefore deduce from (29) that

$$
\mathbb{E}[F \psi(F)]=\mathbb{E}\left[\psi(F) \cdot L L^{-1} F\right]=-\mathbb{E}\left[\Gamma_{0}\left(\psi(F), L^{-1} F\right)\right] .
$$

Now, by the definition of $\Gamma_{0}$ and Lemma 2.7 (b) we obtain that

$$
\begin{aligned}
2 \Gamma_{0}\left(\psi(F), L^{-1} F\right) & \int_{\mathcal{Z}}\left(D_{z}^{+} \psi(F)\right)\left(D_{z}^{+} L^{-1} F\right) \mu(d z)+\int_{\mathcal{Z}}\left(D_{z}^{-} \psi(F)\right)\left(D_{z}^{-} L^{-1} F\right) \eta(d z) \\
= & \psi^{\prime}(F) \int_{\mathcal{Z}}\left(D_{z}^{+} F\right)\left(D_{z}^{+} L^{-1} F\right) \mu(d z) \\
& +\int_{\mathcal{Z}} R_{\psi}^{+}(F, z)\left(D_{z}^{+} F\right)^{2}\left(D_{z}^{+} L^{-1} F\right) \mu(d z) \\
& +\psi^{\prime}(F) \int_{\mathcal{Z}}\left(D_{z}^{-} F\right)\left(D_{z}^{-} L^{-1} F\right) \eta(d z) \\
& +\int_{\mathcal{Z}} R_{\psi}^{-}(F, z)\left(D_{z}^{-} F\right)^{2}\left(D_{z}^{-} L^{-1} F\right) \eta(d z) \\
= & \psi^{\prime}(F) \int_{\mathcal{Z}}\left(D_{z}^{+} F\right)\left(D_{z}^{+} L^{-1} F\right) \mu(d z)+R_{+} \\
& +\psi^{\prime}(F) \int_{\mathcal{Z}}\left(D_{z}^{-} F\right)\left(D_{z}^{-} L^{-1} F\right) \eta(d z)+R_{-} \\
= & 2 \psi^{\prime}(F) \Gamma_{0}\left(F, L^{-1} F\right)+R_{+}+R_{-}
\end{aligned}
$$


with

$$
\begin{aligned}
\mathbb{E}\left|R_{+}\right| & \leq \frac{\left\|\psi^{\prime \prime}\right\|_{\infty}}{2} \mathbb{E}\left[\int_{\mathcal{Z}}\left|D_{z}^{+} F\right|^{2}\left|D_{z}^{+} L^{-1} F\right| \mu(d z)\right] \\
& \leq \mathbb{E}\left[\int_{\mathcal{Z}}\left|D_{z}^{+} F\right|^{2}\left|D_{z}^{+} L^{-1} F\right| \mu(d z)\right]
\end{aligned}
$$

and

$$
\begin{aligned}
\mathbb{E}\left|R_{-}\right| & \leq \frac{\left\|\psi^{\prime \prime}\right\|_{\infty}}{2} \mathbb{E}\left[\int_{\mathcal{Z}}\left|D_{z}^{-} F\right|^{2}\left|D_{z}^{-} L^{-1} F\right| \eta(d z)\right] \\
& \leq \mathbb{E}\left[\int_{\mathcal{Z}}\left|D_{z}^{-} F\right|^{2}\left|D_{z}^{-} L^{-1} F\right| \eta(d z)\right] \\
& =\mathbb{E}\left[\int_{\mathcal{Z}}\left|D_{z}^{+} F\right|^{2}\left|D_{z}^{+} L^{-1} F\right| \mu(d z)\right],
\end{aligned}
$$

where the last identity holds by virtue of (13), as applied to

$$
V(z)=\left(\mathfrak{f}\left(\eta+\delta_{z}\right)-\mathfrak{f}(\eta)\right)^{2}\left|\mathfrak{f}^{*}\left(\eta+\delta_{z}\right)-\mathfrak{f}^{*}(\eta)\right|,
$$

where $\mathfrak{f}$ is a representative of $F$ and $\mathfrak{f}^{*}$ is a representative of $L^{-1} F$. Thus, from (57) and (58) we infer

$$
\begin{aligned}
\left|\mathbb{E}\left[\psi^{\prime}(F)-F \psi(F)\right]\right| \leq & \left|\mathbb{E}\left[\psi^{\prime}(F)\left(1-\Gamma_{0}\left(F,-L^{-1} F\right)\right)\right]\right| \\
& +\frac{1}{2}\left(\mathbb{E}\left|R_{+}\right|+\mathbb{E}\left|R_{-}\right|\right),
\end{aligned}
$$

and from (55), (59), (60) and (61) we conclude that

$$
\begin{aligned}
\left|\mathbb{E}\left[\psi^{\prime}(F)-F \psi(F)\right]\right| \leq & \sqrt{\frac{2}{\pi}} \mathbb{E}\left|1-\Gamma_{0}\left(F,-L^{-1} F\right)\right| \\
& +\mathbb{E}\left[\int_{\mathcal{Z}}\left|D_{z}^{+} F\right|^{2}\left|D_{z}^{+} L^{-1} F\right| \mu(d z)\right] .
\end{aligned}
$$

Plugging such an estimate into (56) yields (52). By (29), we know that

$$
\mathbb{E}\left[\Gamma_{0}\left(F,-L^{-1} F\right)\right]=\operatorname{Var}(F)=\mathbb{E}\left[F^{2}\right]
$$

and hence, (53) follows from (52) by using the triangle and Cauchy-Schwarz inequalities. To prove (54), we first apply the Cauchy-Schwarz inequality to obtain

$$
\int_{\mathcal{Z}} \mathbb{E}\left[\left|D_{z}^{+} F\right|^{3}\right] \mu(d z) \leq\left(\int_{\mathcal{Z}} \mathbb{E}\left[\left|D_{z}^{+} F\right|^{4}\right] \mu(d z)\right)^{1 / 2}\left(\int_{\mathcal{Z}} \mathbb{E}\left[\left|D_{z}^{+} F\right|^{2}\right] \mu(d z)\right)^{1 / 2} \text {. }
$$

But, by using the isometry properties of multiple integrals we have

$$
\begin{aligned}
\int_{\mathcal{Z}} \mathbb{E}\left[\left|D_{z}^{+} F\right|^{2}\right] \mu(d z) & =q^{2} \int_{\mathcal{Z}} \mathbb{E}\left[I_{q-1}(f(z, \cdot))^{2}\right] \mu(d z) \\
& =q^{2}(q-1) ! \int_{\mathcal{Z}}\|f(z, \cdot)\|_{2}^{2} \mu(d z) \\
& =q q !\|f\|_{2}^{2}=q \mathbb{E}\left[F^{2}\right]=q .
\end{aligned}
$$


Hence, we obtain

$$
q^{-1} \int_{\mathcal{Z}} \mathbb{E}\left[\left|D_{z}^{+} F\right|^{3}\right] \mu(d z) \leq \frac{1}{\sqrt{q}}\left(\int_{\mathcal{Z}} \mathbb{E}\left[\left|D_{z}^{+} F\right|^{4}\right] \mu(d z)\right)^{1 / 2}
$$

proving (54).

The next result provides a similar estimate in the Kolmogorov distance.

Proposition 4.3. Under the same assumptions as in Proposition 4.1, one has the bounds

$$
\begin{aligned}
d_{\mathrm{Kol}}(F, N) \leq & \mathbb{E}\left|1-\Gamma_{0}\left(F,-L^{-1} F\right)\right| \\
& +\mathbb{E}\left[(|F|+\sqrt{2 \pi} / 4) \int_{\mathcal{Z}}\left(D_{z}^{+} F\right)^{2}\left|D_{z}^{+} L^{-1} F\right| \mu(d z)\right] \\
& +\sup _{x \in \mathbb{R}} \mathbb{E}\left[\int_{\mathcal{Z}}\left(D_{z}^{+} F\right)\left|D_{z}^{+}\left(L^{-1} F\right)\right| D_{z}^{+} \mathbf{1}_{\{F>x\}} \mu(d z)\right] \\
\leq & \left|1-\mathbb{E}\left[F^{2}\right]\right|+\sqrt{\operatorname{Var}\left(\Gamma_{0}\left(F,-L^{-1} F\right)\right)} \\
& +\mathbb{E}\left[\left(\int_{\mathcal{Z}}\left(D_{z}^{+} F\right)^{2} \mu(d z)\right)^{2}\right]^{1 / 4}\left(1+\mathbb{E}\left[F^{4}\right]^{1 / 4}\right) \\
& \times \sqrt{\mathbb{E}\left[\int_{\mathcal{Z}}\left(D_{z}^{+} F\right)^{2}\left(D_{z}^{+}\left(L^{-1} F\right)\right)^{2} \mu(d z)\right]} \\
& +\sup _{x \in \mathbb{R}} \mathbb{E}\left[\int_{\mathcal{Z}}\left(D_{z}^{+} F\right)\left|D_{z}^{+}\left(L^{-1} F\right)\right| D^{+} \mathbf{1}_{\{F>x\}} \mu(d z)\right] .
\end{aligned}
$$

If $F=I_{q}(f)$ for some $q \geq 1$ and some square-integrable, symmetric kernel $f$ on $\mathcal{Z}^{q}$ and $\mathbb{E}\left[F^{2}\right]=q !\|f\|_{2}^{2}=1$, then (64) becomes

$$
\begin{aligned}
d_{\mathrm{Kol}}(F, N) \leq & \sqrt{\operatorname{Var}\left(q^{-1} \Gamma_{0}(F, F)\right)} \\
& +\frac{1}{q}\left(1+\mathbb{E}\left[F^{4}\right]^{1 / 4}\right) \\
& \times \mathbb{E}\left[\left(\int_{\mathcal{Z}}\left(D_{z}^{+} F\right)^{2} \mu(d z)\right)^{2}\right]^{1 / 4} \sqrt{\mathbb{E}\left[\int_{\mathcal{Z}}\left(D_{z}^{+} F\right)^{4} \mu(d z)\right]} \\
& +\frac{1}{q} \sup _{x \in \mathbb{R}} \mathbb{E}\left[\int_{\mathcal{Z}}\left(D_{z}^{+} F\right)\left|D_{z}^{+} F\right| D_{z}^{+} \mathbf{1}_{\{F>x\}} \mu(d z)\right] .
\end{aligned}
$$

PROOF. Fix $x \in \mathbb{R}$. According to Propositon 6.1, we can write

$$
|\mathbb{P}(F \leq x)-\mathbb{P}(N \leq x)|=\left|\mathbb{E}\left[g_{x}^{\prime}(F)-F g_{x}(F)\right]\right|,
$$


where $g_{x}$ is the solution of the Stein equation (76) associated with $x$, whose properties are stated in Proposition 6.1. Using Proposition 6.1 and reasoning as in the proof of Proposition 4.1, one deduces that

$$
\begin{aligned}
\mid \mathbb{E}\left[g_{x}^{\prime}(F)\right. & \left.-F g_{x}(F)\right] \mid \\
\leq & \mathbb{E}\left[\left|g_{x}^{\prime}(F)\right|\left|1-\Gamma_{0}\left(F,-L^{-1} F\right)\right|\right] \\
& +\frac{1}{4} \mathbb{E}\left[(|F|+\sqrt{2 \pi} / 4) \int_{\mathcal{Z}}\left(D_{z}^{+} F\right)^{2}\left|D_{z}^{+}\left(L^{-1} F\right)\right| \mu(d z)\right] \\
& +\frac{1}{2} \mathbb{E}\left[\int_{\mathcal{Z}}\left(D_{z}^{+} F\right)\left|D_{z}^{+}\left(L^{-1} F\right)\right| D_{z}^{+} \mathbf{1}_{\{F>x\}} \mu(d z)\right] \\
& +\frac{3}{4} \mathbb{E}\left[\int_{\mathcal{Z}}\left(\left|F-D_{z}^{-} F\right|+\sqrt{2 \pi} / 4\right)\left(D_{z}^{-} F\right)^{2}\left|D_{z}^{-}\left(L^{-1} F\right)\right| \eta(d z)\right] \\
& +\frac{1}{2} \mathbb{E}\left[\int_{\mathcal{Z}}\left(D_{z}^{-} F\right)\left|D_{z}^{-}\left(L^{-1} F\right)\right| D_{z}^{-} \mathbf{1}_{\{F>x\}} \eta(d z)\right] .
\end{aligned}
$$

Note that, in order to obtain the previous estimate, one has to use Point (f) and Point $(\mathrm{g})$ in Proposition 6.1, respectively, in order to control the quantities $\left|D_{z}^{+} g_{x}(F)-g_{x}^{\prime}(F) D_{z}^{+} F\right|$ and $\left|D_{z}^{-} g_{x}(F)-g_{x}^{\prime}(F) D_{z}^{-} F\right|$. Bound (63) can now be deduced by applying (13) to the mappings

$$
V(z)=(|\mathfrak{f}(\eta)|+\sqrt{2 \pi} / 4)\left(\mathfrak{f}\left(\eta+\delta_{z}\right)-\mathfrak{f}(\eta)\right)^{2}\left|\mathfrak{f}^{*}\left(\eta+\delta_{z}\right)-\mathfrak{f}^{*}(\eta)\right|,
$$

and

$$
V(z)=\mathbf{1}_{\left\{\mathfrak{f}\left(\eta+\delta_{z}\right)>x\right\}}\left(\mathfrak{f}\left(\eta+\delta_{z}\right)-\mathfrak{f}(\eta)\right)\left|\mathfrak{f}^{*}\left(\eta+\delta_{z}\right)-\mathfrak{f}^{*}(\eta)\right|,
$$

where $\mathfrak{f}$ and $\mathfrak{f}^{*}$ are representatives of $F$ and $L^{-1} F$, respectively. The estimate (64) can be deduced by applying the Cauchy-Schwarz and triangle inequalities to the middle term of (63). The second part of the statement immediately follows from (64) and from the fact that, if $F=I_{q}(f)$, then $-L^{-1} F=q^{-1} F$.

End of The PROOF of TheOrem 1.3. Since, under Assumption A, one has that

$$
\Gamma(F, F)=\Gamma_{0}(F, F) \quad \text { a.s.- } \mathbb{P},
$$

the estimate (3) is a direct consequence of (54), Lemma 3.1 and Lemma 3.2, as well as of elementary simplifications. Similarly, (5) follows from (65), Lemma 3.1, Lemma 3.2 and Lemma 3.3, combined with the estimate

$$
\mathbb{E}\left[\left(\int_{\mathcal{Z}}\left(D_{z}^{+} F\right)^{2} \mu(d z)\right)^{2}\right]^{1 / 4} \leq 4^{1 / 4} \mathbb{E}\left[\left(\Gamma_{0}(F, F)\right)^{2}\right]^{1 / 4} \leq \sqrt{2 q} \mathbb{E}\left[F^{4}\right]^{1 / 4},
$$

where we have used (39). Finally, the bound (6) follows from (5) by distinguishing the cases

$$
\mathbb{E}\left[F^{4}\right]>3+\frac{1}{121} \quad \text { and } \quad \mathbb{E}\left[F^{4}\right] \leq 3+\frac{1}{121}
$$


and by taking into account the fact that the Kolmogorov distance is bounded by 1 .

Proof of Proposition 1.6. Fix $q \geq 2$. Reasoning as in Nualart and Peccati (2005), Corollary 2, if a Gaussian random variable $F:=I_{q}(f) \in C_{q}$ such that $\mathbb{E}\left[I_{q}(f)^{2}\right]:=c>0$ existed, then $\mathbb{E}\left[F^{4}\right]-3 c^{2}=0$. Formulae (43)-(44), together with the explicit form of $D_{q}$ would therefore imply that $f \otimes_{r} f=0$ for every $r=$ $1, \ldots, q-1$, where $q$ is the $r$ th contraction of $f$ with itself, as defined in Nourdin and Peccati (2012), Appendix B. This conclusion contradicts the fact that $c=$ $q !\|f\|_{2}^{2}>0$. The case $q=1$ follows immediately from the relation $\mathbb{E}\left[I_{1}(f)^{4}\right]=$ $3\|f\|_{2}^{4}+\int_{\mathcal{Z}} f^{4} d \mu$.

5. Proof of Theorem 1.7. We begin by giving the analog of Proposition 4.1 for Gamma approximation.

Proposition 5.1. Let $F \in \operatorname{dom} D$ satisfy the same assumptions as in the statement of Proposition 4.1, and let $Z_{v} \sim \bar{\Gamma}(v)$ have the centered Gamma distribution with parameter $v>0$. Then we have the bounds

$$
\begin{aligned}
d_{2}\left(F, Z_{v}\right) \leq & \max \left(1, \frac{2}{v}\right) \mathbb{E}\left|2(F+v)-\Gamma_{0}\left(F,-L^{-1} F\right)\right| \\
& +\max \left(1, \frac{1}{v}+\frac{1}{2}\right) \int_{\mathcal{Z}} \mathbb{E}\left[\left|D_{z}^{+} F\right|^{2}\left|D_{z}^{+} L^{-1} F\right|\right] \mu(d z) \\
\leq & \max \left(1, \frac{2}{v}\right)\left|2 v-\mathbb{E}\left[F^{2}\right]\right| \\
& +\max \left(1, \frac{2}{v}\right) \sqrt{\operatorname{Var}\left(2 F-\Gamma_{0}\left(F,-L^{-1} F\right)\right)} \\
& +\max \left(1, \frac{1}{v}+\frac{1}{2}\right) \int_{\mathcal{Z}} \mathbb{E}\left[\left|D_{z}^{+} F\right|^{2}\left|D_{z}^{+} L^{-1} F\right|\right] \mu(d z) .
\end{aligned}
$$

If, furthermore, $F=I_{q}(f)$ for some $q \geq 1$ and some square-integrable, symmetric kernel $f$ on $\mathcal{Z}^{q}$ and $\mathbb{E}\left[F^{2}\right]=q !\|f\|_{2}^{2}=2 v$, then $-L^{-1} F=q^{-1} F$,

$$
\begin{aligned}
\mathbb{E}\left[\Gamma\left(F,-L^{-1} F\right)-2 F\right] & =q^{-1} \mathbb{E}\left[\Gamma_{0}(F, F)\right]=2 v \quad \text { and } \\
\int_{\mathcal{Z}} \mathbb{E}\left[\left|D_{z}^{+} F\right|^{2}\left|D_{z}^{+} L^{-1} F\right|\right] \mu(d z) & =q^{-1} \int_{\mathcal{Z}} \mathbb{E}\left[\left|D_{z}^{+} F\right|^{3}\right] \mu(d z) \\
& \leq\left(\frac{2 v}{q} \int_{\mathcal{Z}} \mathbb{E}\left[\left|D_{z}^{+} F\right|^{4}\right] \mu(d z)\right)^{1 / 2}
\end{aligned}
$$


so that the previous estimate (67) can be further bounded to give

$$
\begin{aligned}
d_{2}\left(F, Z_{v}\right) \leq & \max \left(1, \frac{2}{v}\right) \sqrt{\operatorname{Var}\left(2 F-q^{-1} \Gamma_{0}(F, F)\right)} \\
& +\max \left(\sqrt{2 v}, \sqrt{\frac{2}{v}}+\sqrt{\frac{v}{2}}\right)\left(\frac{1}{q} \int_{\mathcal{Z}} \mathbb{E}\left[\left|D_{z}^{+} F\right|^{4}\right] \mu(d z)\right)^{1 / 2} .
\end{aligned}
$$

PROOF. Using the recently obtained bounds on the solution to the centered Gamma Stein equation from Theorem 2.3 of Döbler and Peccati (2017b), it is easy to see that

$$
d_{2}\left(F, Z_{v}\right) \leq \sup _{\psi \in \mathcal{F}_{2, v}}\left|\mathbb{E}\left[2(F+v) \psi^{\prime}(F)-F \psi(F)\right]\right|,
$$

where $\mathcal{F}_{2, v}$ denotes the class of all continuously differentiable functions $\psi$ in $\mathbb{R}$ such that both $\psi$ and $\psi^{\prime}$ are Lipschitz-continuous with minimum Lipschitzconstants

$$
\left\|\psi^{\prime}\right\|_{\infty} \leq \max \left(1, \frac{2}{v}\right) \text { and }\left\|\psi^{\prime \prime}\right\|_{\infty} \leq \max \left(2, \frac{1}{v}+1\right) .
$$

The rest of the argument follows a route that is completely analogous to the one leading to the proof of Proposition 4.1; the details are omitted for the sake of conciseness.

LEMMA 5.2. Let $q \geq 1$ be an integer and and consider a random variable $F$ such that $F=I_{q}(f) \in C_{q}=\operatorname{Ker}(L+q I), \mathbb{E}\left[F^{2}\right]=2 v$ and $\mathbb{E}\left[F^{4}\right]<\infty$. Then $F, F^{2} \in \operatorname{dom} L$, and

$$
\begin{aligned}
\operatorname{Var}\left(2 F-q^{-1} \Gamma(F, F)\right)= & \sum_{\substack{1 \leq p \leq 2 q-1: \\
p \neq q}}\left(1-\frac{p}{2 q}\right)^{2} \operatorname{Var}\left(\operatorname{proj}\left\{F^{2} \mid C_{p}\right\}\right) \\
& +\frac{1}{4} \operatorname{Var}\left(\operatorname{proj}\left\{F^{2} \mid C_{q}\right\}-4 F\right) \\
= & \sum_{\substack{1 \leq p \leq 2 q-1: \\
p \neq q}}\left(1-\frac{p}{2 q}\right)^{2} \operatorname{Var}\left(\operatorname{proj}\left\{F^{2} \mid C_{p}\right\}\right) \\
& +\frac{1}{4} \operatorname{Var}\left(\operatorname{proj}\left\{F^{2} \mid C_{q}\right\}\right)+8 v-2 \mathbb{E}\left[F^{3}\right]=V_{1}+V_{2},
\end{aligned}
$$

where we define

(69) $\quad V_{1}:=\sum_{\substack{1 \leq p \leq 2 q-1: \\ p \neq q}}\left(1-\frac{p}{2 q}\right)^{2} \operatorname{Var}\left(\operatorname{proj}\left\{F^{2} \mid C_{p}\right\}\right)$ and

(70) $V_{2}:=\frac{1}{4} \operatorname{Var}\left(\operatorname{proj}\left\{F^{2} \mid C_{q}\right\}\right)+8 v-2 \mathbb{E}\left[F^{3}\right]=\frac{1}{4} \operatorname{Var}\left(\operatorname{proj}\left\{F^{2} \mid C_{q}\right\}-4 F\right)$. 
PROOF. The first identity easily follows from (42) and the orthogonality of the chaos decomposition. The second one follows from this and the formula

$$
\operatorname{Var}(X+Y)=\operatorname{Var}(X)+\operatorname{Var}(Y)+2 \operatorname{Cov}(X, Y)
$$

upon observing that

$$
\operatorname{Cov}\left(\operatorname{proj}\left\{F^{2} \mid C_{q}\right\},-4 F\right)=-4 \mathbb{E}\left[F^{3}\right],
$$

again by orthogonality.

LEMMA 5.3. Let $q \geq 1$ be an integer and let $F \in L^{4}(\mathbb{P})$ be an element of the qth Wiener chaos $C_{q}$, such that $F$ verifies Assumption $\mathrm{A}$ and $\mathbb{E}\left[F^{2}\right]=2 v$. The following relations are in order:

$$
\begin{aligned}
\frac{1}{6 q}(\mathbb{E} & {\left.\left[F^{4}\right]-12 \mathbb{E}\left[F^{3}\right]-12 v^{2}+48 v\right)+\frac{1}{12 q^{2}} \int_{\mathcal{Z}} \mathbb{E}\left[\left|D_{z}^{+} F\right|^{4}\right] \mu(d z) } \\
& \leq \operatorname{Var}\left(2 F-q^{-1} \Gamma(F, F)\right)=\operatorname{Var}\left(2 F-q^{-1} \Gamma_{0}(F, F)\right) \\
& \leq \frac{1}{3}\left(\mathbb{E}\left[F^{4}\right]-12 \mathbb{E}\left[F^{3}\right]-12 v^{2}+48 v\right)+\frac{1}{6 q} \int_{\mathcal{Z}} \mathbb{E}\left[\left|D_{z}^{+} F\right|^{4}\right] \mu(d z) .
\end{aligned}
$$

Proof. Recall that, under the assumptions in the statement, $\Gamma(F, F)=$ $\Gamma_{0}(F, F)$. Using orthogonality, from Lemma 3.2 and (42) we obtain

$$
\begin{aligned}
\mathbb{E}\left[F^{4}\right]= & \frac{3}{q} \mathbb{E}\left[F^{2} \Gamma(F, F)\right]-\frac{1}{2 q} \int_{\mathcal{Z}} \mathbb{E}\left[\left|D_{z}^{+} F\right|^{4}\right] \mu(d z) \\
= & 3\left(\mathbb{E}\left[F^{2}\right]\right)^{2}+3 \sum_{p=1}^{2 q}\left(1-\frac{p}{2 q}\right) \operatorname{Var}\left(\operatorname{proj}\left\{F^{2} \mid C_{p}\right\}\right) \\
& -\frac{1}{2 q} \int_{\mathcal{Z}} \mathbb{E}\left[\left|D_{z}^{+} F\right|^{4}\right] \mu(d z) \\
= & 12 v^{2}+3 \sum_{1 \leq p \leq 2 q-1:}\left(1-\frac{p}{2 q}\right) \operatorname{Var}\left(\operatorname{proj}\left\{F^{2} \mid C_{p}\right\}\right) \\
& +\frac{3}{2} \operatorname{Var}\left(\operatorname{proj}\left\{F^{2} \mid C_{q}\right\}\right)-\frac{1}{2 q} \int_{\mathcal{Z}} \mathbb{E}\left[\left|D_{z}^{+} F\right|^{4}\right] \mu(d z) .
\end{aligned}
$$

Hence, recalling the definition of $V_{2}$ in (70) we conclude from Lemma 5.2 that

$$
\begin{aligned}
\mathbb{E}\left[F^{4}\right]-12 \mathbb{E}\left[F^{3}\right]-12 v^{2}+48 v & \\
=3 & \sum_{\substack{1 \leq p \leq 2 q-1: \\
p \neq q}}\left(1-\frac{p}{2 q}\right) \operatorname{Var}\left(\operatorname{proj}\left\{F^{2} \mid C_{p}\right\}\right)-\frac{1}{2 q} \int_{\mathcal{Z}} \mathbb{E}\left[\left|D_{z}^{+} F\right|^{4}\right] \mu(d z)
\end{aligned}
$$


1908

$$
\begin{aligned}
& +\frac{3}{2} \operatorname{Var}\left(\operatorname{proj}\left\{F^{2} \mid C_{q}\right\}\right)-12 \mathbb{E}\left[F^{3}\right]+48 v \\
& =3 \sum_{\substack{1 \leq p \leq 2 q-1: \\
p \neq q}}\left(1-\frac{p}{2 q}\right) \operatorname{Var}\left(\operatorname{proj}\left\{F^{2} \mid C_{p}\right\}\right) \\
& +6 V_{2}-\frac{1}{2 q} \int_{\mathcal{Z}} \mathbb{E}\left[\left|D_{z}^{+} F\right|^{4}\right] \mu(d z) .
\end{aligned}
$$

Now, recalling also the definition (69) of $V_{1}$ and using the simple chain of inequalities

$$
\left(1-\frac{p}{2 q}\right)^{2} \leq\left(1-\frac{p}{2 q}\right) \leq 2 q\left(1-\frac{p}{2 q}\right)^{2}, \quad 1 \leq p \leq 2 q-1,
$$

we obtain on the one hand that

$$
\begin{aligned}
\mathbb{E}\left[F^{4}\right] & -12 \mathbb{E}\left[F^{3}\right]-12 v^{2}+48 v \\
\geq & 3 \sum_{\substack{1 \leq p \leq 2 q-1: \\
p \neq q}}\left(1-\frac{p}{2 q}\right)^{2} \operatorname{Var}\left(\operatorname{proj}\left\{F^{2} \mid C_{p}\right\}\right) \\
& +6 V_{2}-\frac{1}{2 q} \int_{\mathcal{Z}} \mathbb{E}\left[\left|D_{z}^{+} F\right|^{4}\right] \mu(d z) \\
= & 3 V_{1}+6 V_{2}-\frac{1}{2 q} \int_{\mathcal{Z}} \mathbb{E}\left[\left|D_{z}^{+} F\right|^{4}\right] \mu(d z) \\
\geq & 3 \operatorname{Var}\left(2 F-q^{-1} \Gamma(F, F)\right)-\frac{1}{2 q} \int_{\mathcal{Z}} \mathbb{E}\left[\left|D_{z}^{+} F\right|^{4}\right] \mu(d z),
\end{aligned}
$$

and, on the other hand,

$$
\begin{aligned}
\mathbb{E}\left[F^{4}\right] & -12 \mathbb{E}\left[F^{3}\right]-12 v^{2}+48 v \\
\leq & 6 q \sum_{\substack{1 \leq p \leq 2 q-1 \\
p \neq q}}\left(1-\frac{p}{2 q}\right)^{2} \operatorname{Var}\left(\operatorname{proj}\left\{F^{2} \mid C_{p}\right\}\right) \\
& +6 V_{2}-\frac{1}{2 q} \int_{\mathcal{Z}} \mathbb{E}\left[\left|D_{z}^{+} F\right|^{4}\right] \mu(d z) \\
\leq & 6 q \operatorname{Var}\left(2 F-q^{-1} \Gamma(F, F)\right)-\frac{1}{2 q} \int_{\mathcal{Z}} \mathbb{E}\left[\left|D_{z}^{+} F\right|^{4}\right] \mu(d z)
\end{aligned}
$$

The statement of the lemma now follows from (71) and (72).

END OF THE PROOF OF THEOREM 1.7. The claim of Theorem 1.7 is now an immediate consequence of the bound (68) and of the upper bound given in Lemma 5.3. 


\section{Proofs of technical lemmas.}

6.1. Proof of Lemma 2.7. We first prove part (a). We just prove (31) and (32), since the derivation of (33) and (34) is very similar. Let $f$ be a representative for $F$, that is, $F=f(\eta)$. Then, by the binomial identity, we have

$$
\begin{aligned}
\left(D_{z}^{+} F\right)^{2} & =\left(f\left(\eta+\delta_{z}\right)-f(\eta)\right)^{2} \\
& =f\left(\eta+\delta_{z}\right)^{2}-f(\eta)^{2}-2 f\left(\eta+\delta_{z}\right) f(\eta)+2 f(\eta)^{2} \\
& =D_{z}^{+} F^{2}-2 f(\eta)\left(f\left(\eta+\delta_{z}\right)-f(\eta)\right)=D_{z}^{+} F^{2}-2 F D_{z}^{+} F
\end{aligned}
$$

such that (31) holds true. Similarly, using (31), we obtain

$$
\begin{aligned}
\left(D_{z}^{+} F\right)^{3}= & \left(f\left(\eta+\delta_{z}\right)-f(\eta)\right)^{3}=f\left(\eta+\delta_{z}\right)^{3}-f(\eta)^{3}-3 f\left(\eta+\delta_{z}\right)^{2} f(\eta) \\
& +3 f\left(\eta+\delta_{z}\right) f(\eta)^{2} \\
= & D_{z}^{+} F^{3}+3 f(\eta)^{2}\left(f\left(\eta+\delta_{z}\right)-f(\eta)\right)-3 f(\eta)\left(f\left(\eta+\delta_{z}\right)^{2}-f(\eta)^{2}\right) \\
= & D_{z}^{+} F^{3}+3 F^{2} D_{z}^{+} F-3 F D_{z}^{+} F^{2} \\
= & D_{z}^{+} F^{3}+3 F^{2} D_{z}^{+} F-3 F\left(D_{z}^{+} F\right)^{2}-6 F^{2} D_{z}^{+} F \\
= & D_{z}^{+} F^{3}-3 F^{2} D_{z}^{+} F-3 F\left(D_{z}^{+} F\right)^{2}
\end{aligned}
$$

which is equivalent to (32). Now we turn to the proof of (b). Again, we just prove the part involving $D_{z}^{+}$. By a suitable version of Taylor's formula, for $x, y \in \mathbb{R}$ we have

$$
\psi(y)=\psi(x)+\psi^{\prime}(x)(y-x)+R_{\psi}(x, y)(y-x)^{2},
$$

where

$$
\left|R_{\psi}(x, y)\right| \leq \frac{\left\|\psi^{\prime \prime}\right\|_{\infty}}{2} .
$$

Now the result follows by letting $x=F=f(\eta), y=f\left(\eta+\delta_{z}\right)$ and $R_{\psi}^{+}(F, z)=$ $R_{\psi}\left(f(\eta), f\left(\eta+\delta_{z}\right)\right)$.

6.2. Proof of Lemma 2.4. The method of proof we apply is similar to the one used for the proof of Proposition 5 in Last (2016), which gives the product formula for multiple Wiener-Itô integrals. Let

$$
F G=\mathbb{E}[F G]+\sum_{m=1}^{\infty} I_{m}\left(h_{m}\right)
$$

denote the chaos decomposition of $F G$. Let us, for the moment, only assume that $F=I_{p}(f), G=I_{q}(g) \in L^{2}(\mathbb{P})$. We will prove the following statements $(\tilde{a})$ and 
( $\tilde{b})$ simultaneously by induction on $k:=p+q \geq 2$ :
(a) $\frac{1}{m !} \mathbb{E}\left[D_{z_{1}, \ldots, z_{m}}^{(m)}(F G)\right]=0 \quad$ for all $m>k$ and $z_{1}, \ldots, z_{m} \in \mathcal{Z}$,
(ש) $\frac{1}{k !} \mathbb{E}\left[D_{z_{1}, \ldots, z_{k}}^{(k)}(F G)\right]=f \tilde{\otimes} g\left(z_{1}, \ldots, z_{k}\right) \quad$ for all $z_{1}, \ldots, z_{k} \in \mathcal{Z}$.

Once this is shown, if $F, G \in L^{4}(\mathbb{P})$ such that $F G$ has a chaotic decomposition (73), (a) and (b) immediately follow from $(\tilde{a})$ and $(\tilde{b})$, respectively, by virtue of (17). If $k=2$, then necessarily $p=q=1$ and, by (35) and since $F, G \in \operatorname{dom} D$, for all $y, z \in \mathcal{Z}$ we have

$$
\begin{aligned}
D_{z}^{+}(F G) & =f(z) I_{1}(g)+g(z) I_{1}(f)+f(z) g(z) \quad \text { and } \\
D_{y, z}^{(2)}(F G) & =f(z) g(y)+f(y) g(z)=2 f \tilde{\otimes} g(y, z) .
\end{aligned}
$$

This immediately implies that $D^{(m)}(F G)=0$ for all $m>2$. We can thus infer that

$$
\frac{1}{2} \mathbb{E}\left[D_{z_{1}, z_{2}}^{(2)}(F G)\right]=f \tilde{\otimes} g\left(z_{1}, z_{2}\right) \quad \text { and } \quad \frac{1}{m !} \mathbb{E}\left[D_{z_{1}, \ldots, z_{m}}^{(m)}(F G)\right]=0
$$

for all $m>2$ and $z_{1}, \ldots, z_{m} \in \mathcal{Z}$. Thus, $(\tilde{a})$ and $(\tilde{b})$ hold true for $k=2$. Now assume that $k>2$. Again, since $F, G \in \operatorname{dom} D$, from (35) we obtain that

$$
\begin{aligned}
D_{z_{k}}^{+}(F G)= & p I_{q}(g) I_{p-1}\left(f\left(z_{k}, \cdot\right)\right)+q I_{p}(f) I_{q-1}\left(g\left(z_{k}, \cdot\right)\right) \\
& +p q I_{p-1}\left(f\left(z_{k}, \cdot\right)\right) I_{q-1}\left(g\left(z_{k}, \cdot\right)\right) \\
= & : p \tilde{F}_{z_{k}} G+q \tilde{G}_{z_{k}} F+p q \tilde{F}_{z_{k}} \tilde{G}_{z_{k}}
\end{aligned}
$$

holds for all $z_{k} \in \mathcal{Z}$, where $\tilde{F}_{z_{k}}$ and $\tilde{G}_{z_{k}}$ are multiple integrals of orders $p-1$ and $q-1$, respectively. Hence, by the induction hypothesis for claim $(\tilde{a})$ we already conclude that

$$
\mathbb{E}\left[D_{z_{1}, \ldots, z_{k-1}}^{(k-1)}\left(\tilde{F}_{z k} \tilde{G}_{z_{k}}\right)\right]=0
$$

so that

$$
\mathbb{E}\left[D_{z_{1}, \ldots, z_{k}}^{(k)}(F G)\right]=p \mathbb{E}\left[D_{z_{1}, \ldots, z_{k-1}}^{(k-1)}\left(\tilde{F}_{z_{k}} G\right)\right]+q \mathbb{E}\left[D_{z_{1}, \ldots, z_{k-1}}^{(k-1)}\left(F \tilde{G}_{z_{k}}\right)\right] .
$$

By the induction hypothesis for claim $(\tilde{b})$, we have

$$
\begin{aligned}
& \mathbb{E}\left[D_{z_{1}, \ldots, z_{k-1}}^{(k-1)}\left(\tilde{F}_{z_{k}} G\right)\right]=(k-1) !\left(f\left(z_{k}, \cdot\right) \tilde{\otimes} g\right)\left(z_{1}, \ldots, z_{k-1}\right) \quad \text { and } \\
& \mathbb{E}\left[D_{z_{1}, \ldots, z_{k-1}}^{(k-1)}\left(F \tilde{G}_{z_{k}}\right)\right]=(k-1) !\left(f \tilde{\otimes}\left(g\left(z_{k}, \cdot\right)\right)\left(z_{1}, \ldots, z_{k-1}\right)\right.
\end{aligned}
$$

and, in order to prove $(\tilde{b})$, it remains to show that

$$
\begin{aligned}
k !(f \tilde{\otimes} g)\left(z_{1}, \ldots, z_{k}\right)= & p(k-1) !\left(f\left(z_{k}, \cdot\right) \tilde{\otimes} g\right)\left(z_{1}, \ldots, z_{k-1}\right) \\
& +q(k-1) !\left(f \tilde{\otimes}\left(g\left(z_{k}, \cdot\right)\right)\left(z_{1}, \ldots, z_{k-1}\right) .\right.
\end{aligned}
$$


This, however, follows from

$$
\begin{aligned}
k !(f \tilde{\otimes} & g)\left(z_{1}, \ldots, z_{k}\right) \\
= & \sum_{\pi \in \mathbb{S}_{p+q}} f\left(z_{\pi(1)}, \ldots, z_{\pi(p)}\right) g\left(z_{\pi(p+1)}, \ldots, z_{\pi(p+q)}\right) \\
= & \sum_{\pi: k \in\{\pi(1), \ldots, \pi(p)\}} f\left(z_{\pi(1)}, \ldots, z_{\pi(p)}\right) g\left(z_{\pi(p+1)}, \ldots, z_{\pi(p+q)}\right) \\
& +\sum_{\pi: k \notin\{\pi(1), \ldots, \pi(p)\}} f\left(z_{\pi(1)}, \ldots, z_{\pi(p)}\right) g\left(z_{\pi(p+1)}, \ldots, z_{\pi(p+q)}\right) \\
\stackrel{!}{=} & p \sum_{\tau \in \mathbb{S}_{p+q-1}} f\left(z_{k}, z_{\tau(1)}, \ldots, z_{\tau(p-1)}\right) g\left(z_{\tau(p)}, \ldots, z_{\tau(p+q-1)}\right) \\
& +q \sum_{\tau \in \mathbb{S}_{p+q-1}} f\left(z_{\tau(1)}, \ldots, z_{\tau(p)}\right) g\left(z_{k}, z_{\tau(p+1)}, \ldots, z_{\tau(p+q-1)}\right) \\
= & p(k-1) !\left(f\left(z_{k}, \cdot\right) \tilde{\otimes} g\right)\left(z_{1}, \ldots, z_{k-1}\right) \\
& +q(k-1) !\left(f \tilde{\otimes}\left(g\left(z_{k}, \cdot\right)\right)\left(z_{1}, \ldots, z_{k-1}\right) .\right.
\end{aligned}
$$

We explain the identity involving ! in some more detail. Consider the first sum appearing there and note that

$$
\begin{aligned}
& \sum_{\pi: k \in\{\pi(1), \ldots, \pi(p)\}} f\left(z_{\pi(1)}, \ldots, z_{\pi(p)}\right) g\left(z_{\pi(p+1)}, \ldots, z_{\pi(p+q)}\right) \\
= & \sum_{j=1}^{p} \sum_{\pi: \pi(j)=k} f\left(z_{\pi(1)}, \ldots, z_{\pi(j-1)}, z_{k}, z_{\pi(j+1)}, \ldots, z_{\pi(p)}\right) \\
& \times g\left(z_{\pi(p+1)}, \ldots, z_{\pi(p+q)}\right) \\
= & p \sum_{\pi: \pi(1)=k} f\left(z_{k}, z_{\pi(2)}, \ldots, z_{\pi(p)}\right) g\left(z_{\pi(p+1)}, \ldots, z_{\pi(p+q)}\right),
\end{aligned}
$$

where we have used the symmetry of the kernel $f$ to obtain the last identity. Now, since the mapping

$$
\begin{aligned}
\Psi: \mathbb{S}_{k-1} \rightarrow\left\{\pi \in \mathbb{S}_{k}: \pi(1)=k\right\}, \\
\Psi(\sigma)(j):= \begin{cases}k, & j=1 \\
\sigma(j-1), & j \in\{2, \ldots, k\}\end{cases}
\end{aligned}
$$


is a bijection, we obtain that

$$
\begin{aligned}
& \sum_{\pi: \pi(1)=k} f\left(z_{k}, z_{\pi(2)}, \ldots, z_{\pi(p)}\right) g\left(z_{\pi(p+1)}, \ldots, z_{\pi(p+q)}\right) \\
& \quad=\sum_{\tau \in \mathbb{S}_{p+q-1}} f\left(z_{k}, z_{\tau(1)}, \ldots, z_{\tau(p-1)}\right) g\left(z_{\tau(p)}, \ldots, z_{\tau(p+q-1)}\right)
\end{aligned}
$$

proving the claim. Thus, we have proved $(\tilde{b})$.

If $m>k$ and $z_{1}, \ldots, z_{m} \in \mathcal{Z}$, then, by the induction hypothesis on $(\tilde{a})$ and from (74) we obtain

$$
\begin{aligned}
\mathbb{E}\left[D_{z_{1}, \ldots, z_{m}}^{(m)}(F G)\right]= & p \mathbb{E}\left[D_{z_{1}, \ldots, z_{m-1}}^{(m-1)}\left(\tilde{F}_{z_{m}} G\right)\right]+q \mathbb{E}\left[D_{z_{1}, \ldots, z_{m-1}}^{(m-1)}\left(F \tilde{G}_{z_{m}}\right)\right] \\
& +p q \mathbb{E}\left[D_{z_{1}, \ldots, z_{m-1}}^{(m-1)}\left(\tilde{F}_{z_{m}} \tilde{G}_{z_{m}}\right)\right] \\
= & 0
\end{aligned}
$$

for all $z_{1}, \ldots, z_{m} \in \mathcal{Z}$, proving $(\tilde{a})$.

6.3. Stein's equation in the Kolmogorov distance. In order to deal with bounds in the Kolmogorov distance involving remove-one cost operators, we need the following result, containing several estimates on the solution of the Stein's equation associated with test functions having the form of indicators of half-lines. Points (a)-(f) are well known. Point (g) is standard but not explicitly stated in the literature (to our knowledge); a proof is provided for the sake of completeness.

PROPOSITION 6.1. Let $N \sim N(0,1)$ be a centred Gaussian random variable with unit variance and, for every $x \in \mathbb{R}$, introduce the Stein's equation

$$
g^{\prime}(w)-w g(w)=\mathbf{1}_{\{w \leq x\}}-\mathbb{P}(N \leq x),
$$

where $w \in \mathbb{R}$. Then, for every real $x$, there exists a function $g_{x}: \mathbb{R} \rightarrow \mathbb{R}: w \mapsto$ $g_{x}(w)$ satisfying the following properties $(\mathrm{a})-(\mathrm{g})$ :

(a) $g_{x}$ is continuous at every point $w \in \mathbb{R}$, and infinitely differentiable at every $w \neq x$

(b) $g_{x}$ satisfies the relation (76), for every $w \neq x$;

(c) $0<g_{x} \leq \frac{\sqrt{2 \pi}}{4}$;

(d) for every $u, v, w \in \mathbb{R}$,

$$
\left|(w+u) g_{x}(w+u)-(w+v) g_{x}(w+v)\right| \leq\left(|w|+\frac{\sqrt{2 \pi}}{4}\right)(|u|+|v|) ;
$$

(e) adopting the convention

$$
g_{x}^{\prime}(x):=x g_{x}(x)+1-\mathbb{P}(N \leq x),
$$

one has that $\left|g_{x}^{\prime}(w)\right| \leq 1$, for every real $w$; 
(f) using again the convention (78), for all $w, h \in \mathbb{R}$ one has that

$$
\begin{aligned}
\left|g_{x}(w+h)-g_{x}(w)-g_{x}^{\prime}(w) h\right| \leq & \frac{|h|^{2}}{2}\left(|w|+\frac{\sqrt{2 \pi}}{4}\right) \\
& +|h|\left(\mathbf{1}_{[w, w+h)}(x)+\mathbf{1}_{[w+h, w)}(x)\right) \\
= & \frac{|h|^{2}}{2}\left(|w|+\frac{\sqrt{2 \pi}}{4}\right) \\
& +h\left(\mathbf{1}_{[w, w+h)}(x)-\mathbf{1}_{[w+h, w)}(x)\right)
\end{aligned}
$$

(g) under (78), for every $w, h \in \mathbb{R}$ one has that

$$
\begin{aligned}
\left|g_{x}(w)-g_{x}(w-h)-g_{x}^{\prime}(w) h\right| \leq & \frac{3|h|^{2}}{2}\left(|w-h|+\frac{\sqrt{2 \pi}}{4}\right) \\
& +|h|\left(\mathbf{1}_{[w-h, w)}(x)+\mathbf{1}_{[w, w-h)}(x)\right) \\
= & \frac{3|h|^{2}}{2}\left(|w-h|+\frac{\sqrt{2 \pi}}{4}\right) \\
& +h\left(\mathbf{1}_{[w-h, w)}(x)-\mathbf{1}_{[w, w-h)}(x)\right) .
\end{aligned}
$$

Proof. The content of points (a)-(f) is well known; see, for example, Bourguin and Peccati (2016), Section 2.2.2, and the references therein. To show (g), fix $x \in \mathbb{R}$, recall (78) and write, for every $w, h \in \mathbb{R}$,

$$
g_{x}(w)-g_{x}(w-h)-h g_{x}^{\prime}(w)=\int_{0}^{h}\left(g_{x}^{\prime}(w-h+u)-g_{x}^{\prime}(w)\right) d u .
$$

Since $g_{x}$ is a solution of (76) for every real $w$, we have that, for all $w, h \in \mathbb{R}$,

$$
\begin{aligned}
g_{x}(w) & -g_{x}(w-h)-h g_{x}^{\prime}(w) \\
= & \int_{0}^{h}\left((w-h+u) g_{x}(w-h+u)-w g_{x}(w)\right) d u \\
& +\int_{0}^{h}\left(\mathbf{1}_{\{w-h+u \leq x\}}-\mathbf{1}_{\{w \leq x\}}\right) d u \\
:= & J_{1}+J_{2} .
\end{aligned}
$$

It follows that, by the triangle inequality,

$$
\left|g_{x}(w)-g_{x}(w-h)-h g_{x}^{\prime}(x)\right| \leq\left|J_{1}\right|+\left|J_{2}\right| .
$$

Using (77), we have

$$
\left|J_{1}\right| \leq \int_{0}^{h}\left(|w-h|+\frac{\sqrt{2 \pi}}{4}\right)(|u|+|h|) d u=\frac{3 h^{2}}{2}\left(|w-h|+\frac{\sqrt{2 \pi}}{4}\right) .
$$


On the other hand, we have that

$$
\begin{aligned}
\left|J_{2}\right|= & \mathbf{1}_{\{h<0\}}\left|\int_{0}^{h}\left(\mathbf{1}_{\{w-h+u \leq x\}}-\mathbf{1}_{\{w \leq x\}}\right) d u\right| \\
& +\mathbf{1}_{\{h \geq 0\}}\left|\int_{0}^{h}\left(\mathbf{1}_{\{w-h+u \leq x\}}-\mathbf{1}_{\{w \leq x\}}\right) d u\right| \\
= & \mathbf{1}_{\{h<0\}} \int_{h}^{0} \mathbf{1}_{\{w \leq x<w-h+u\}} d u+\mathbf{1}_{\{h \geq 0\}} \int_{0}^{h} \mathbf{1}_{\{w-h+u \leq x<w\}} d u .
\end{aligned}
$$

As a consequence,

$$
\begin{aligned}
\left|J_{2}\right| & \leq \mathbf{1}_{\{h<0\}}(-h) \mathbf{1}_{[w, w-h)}(x)+\mathbf{1}_{\{h \geq 0\}} h \mathbf{1}_{[w-h, w)}(x) \\
& =h\left(\mathbf{1}_{[w-h, w)}(x)-\mathbf{1}_{[w, w-h)}(x)\right) \\
& \left.=|h| \mathbf{1}_{[w-h, w)}(x)+\mathbf{1}_{[w, w-h)}(x)\right) .
\end{aligned}
$$

Using (84) and (85) in (83) yields the conclusion.

Acknowledgments. The authors would like to thank the two anonymous referees, the Associate Editor and Guangqu Zheng for insightful remarks and comments. We are also grateful to Solesne Bourguin, Simon Campese, Günter Last and Matthias Schulte for useful discussions.

\section{REFERENCES}

Azmoodeh, E., Campese, S. and Poly, G. (2014). Fourth Moment Theorems for Markov diffusion generators. J. Funct. Anal. 266 2341-2359. MR3150163

Azmoodeh, E., Malicet, D., Muoule, G. and Poly, G. (2016). Generalization of the NualartPeccati criterion. Ann. Probab. 44 924-954. MR3050508

Bachmann, S. and PECCATI, G. (2016). Concentration bounds for geometric Poisson functionals: Logarithmic Sobolev inequalities revisited. Electron. J. Probab. 21 no. 6, 21. MR3035760

BAKRY, D., Gentil, I. and LedouX, M. (2014). Analysis and Geometry of Markov Diffusion Operators. Grundlehren der Mathematischen Wissenschaften [Fundamental Principles of Mathematical Sciences] 348. Springer, Cham. MR3155209

Bouleau, N. and Denis, L. (2015). Dirichlet Forms Methods for Poisson Point Measures and Lévy Processes. Springer, Cham. MR3444890

Bourguin, S. and Peccati, G. (2014). Semicircular limits on the free Poisson chaos: Counterexamples to a transfer principle. J. Funct. Anal. 267 963-997. MR3150163

Bourguin, S. and Peccati, G. (2016). The Malliavin-Stein method on the Poisson space. In Stochastic Analysis for Poisson Point Processes. Bocconi Springer Ser. 7 185-228. Bocconi Univ. Press. MR3585401

Chen, L. H. Y., Goldstein, L. and Shao, Q.-M. (2011). Normal Approximation by Stein's Method. Springer, Heidelberg. MR2732624

Chen, L. H. Y. and Poly, G. (2015). Stein's method, Malliavin calculus, Dirichlet forms and the fourth moment theorem. In Festschrift Masatoshi Fukushima. Interdiscip. Math. Sci. 17 107-130. World Sci. Publ., Hackensack, NJ. MR3379337

DE JONG, P. (1987). A central limit theorem for generalized quadratic forms. Probab. Theory Related Fields 75 261-277. MR885466 
DE Jong, P. (1989). Central Limit Theorems for Generalized Multilinear Forms. CWI Tract 61. Stichting Mathematisch Centrum, Centrum voor Wiskunde en Informatica, Amsterdam. MR1002734

DE JONG, P. (1990). A central limit theorem for generalized multilinear forms. J. Multivariate Anal. 34 275-289. MR1073110

DöBler, C. and KROKOWSKI, K. (2017). On the fourth moment condition for Rademacher chaos. Ann. Inst. Henri Poincaré Probab. Stat. To appear. arXiv:1706.00751.

Döbler, C. and PeCCATi, G. (2017a). Quantiative de Jong theorems in any dimension. Electron. J. Probab. 22 1-35.

Döbler, C. and Peccati, G. (2017b). The Gamma Stein equation and non-central de Jong theorems. Bernoulli. To appear. arXiv:1612.02279.

Döbler, C., Vidotto, A. and Zheng, G. (2017). Fourth moment theorems on the Poisson space in any dimension. arXiv:1707.01889.

EICHELSBACHER, P. and ThÄLE, C. (2014). New Berry-Esseen bounds for non-linear functionals of Poisson random measures. Electron. J. Probab. 19 no. 102, 25. MR3275854

FISSLER, T. and THÄLE, C. (2016). A four moments theorem for gamma limits on a Poisson chaos. ALEA Lat. Am. J. Probab. Math. Stat. 13 163-192. MR3476211

LACHIÈZE-REY, R. and PECCATI, G. (2013). Fine Gaussian fluctuations on the Poisson space, I: Contractions, cumulants and geometric random graphs. Electron. J. Probab. 18 no. 32, 32. MR3035760

LACHIÈZE-REY, R. and REITZNER, M. (2016). U-statistics in stochastic geometry. In Stochastic Analysis for Poisson Point Processes (G. Peccati and M. Reitzner, eds.). 229-253. Bocconi Univ. Press. MR3585402

LACHIÈZE-REY, R., Schulte, M. and YuKiCH, J. (2016). Normal approximation for sums of stabilizing functionals. Preprint. arXiv:1702.00726.

LAST, G. (2016). Stochastic analysis for Poisson processes. In Stochastic Analysis for Poisson Point Processes. Bocconi Springer Ser. 7 1-36. Bocconi Univ. Press. MR3585396

LAst, G., Peccati, G. and Schulte, M. (2016). Normal approximation on Poisson spaces: Mehler's formula, second order Poincaré inequalities and stabilization. Probab. Theory Related Fields 165 667-723. MR3520016

LAst, G. and Penrose, M. D. (2011). Poisson process Fock space representation, chaos expansion and covariance inequalities. Probab. Theory Related Fields 150 663-690. MR2824870

Last, G. and Penrose, M. (2017). Lectures on the Poisson Process. Cambridge Univ. Press, Cambridge.

Ledoux, M. (2012). Chaos of a Markov operator and the fourth moment condition. Ann. Probab. 40 2439-2459. MR3050508

Ledoux, M., Nourdin, I. and Peccati, G. (2015). Stein's method, logarithmic Sobolev and transport inequalities. Geom. Funct. Anal. 25 256-306. MR3320893

MECKE, J. (1967). Stationäre zufällige Masse auf lokalkompakten Abelschen Gruppen. Z. Wahrsch. Verw. Gebiete 9 36-58. MR0228027

Nourdin, I. and PecCATI, G. (2009a). Stein's method on Wiener chaos. Probab. Theory Related Fields 145 75-118. MR2520122

Nourdin, I. and PeCCATi, G. (2009b). Noncentral convergence of multiple integrals. Ann. Probab. 37 1412-1426. MR2546749

Nourdin, I. and PeCCATI, G. (2012). Normal Approximations with Malliavin Calculus: From Stein's Method to Universality. Cambridge Tracts in Mathematics 192. Cambridge Univ. Press, Cambridge. MR2962301

Nualart, D. and PeCCATi, G. (2005). Central limit theorems for sequences of multiple stochastic integrals. Ann. Probab. 33 177-193. MR2118863

Peccati, G. and ReItZner, M. (2016). Stochastic Analysis for Poisson Point Processes. Springer, Cham. MR3444831 
Peccati, G. and Taqqu, M. S. (2008). Central limit theorems for double Poisson integrals. Bernoulli 14 791-821. MR2537812

Peccati, G. and ThäLe, C. (2013). Gamma limits and $U$-statistics on the Poisson space. ALEA Lat. Am. J. Probab. Math. Stat. 10 525-560. MR3083936

Peccati, G. and Zheng, C. (2010). Multi-dimensional Gaussian fluctuations on the Poisson space. Electron. J. Probab. 15 1487-1527. MR2727319

PeCCATi, G. and Zheng, C. (2014). Universal Gaussian fluctuations on the discrete Poisson chaos. Bernoulli 20 697-715. MR3178515

Peccati, G., Solé, J. L., TAqQU, M. S. and Utzet, F. (2010). Stein's method and normal approximation of Poisson functionals. Ann. Probab. 38 443-478. MR2642882

Reitzner, M. and Schulte, M. (2013). Central limit theorems for U-statistics of Poisson point processes. Ann. Probab. 41 3879-3909.

SCHulte, M. (2016). Normal approximation of Poisson functionals in Kolmogorov distance. $J$. Theoret. Probab. 29 96-117. MR3463079

Schulte, M. and Thäle, C. (2016). Poisson point process convergence and extreme values in stochastic geometry. In Stochastic Analysis for Poisson Point Processes. Bocconi Springer Ser. 7 255-294. Bocconi Univ. Press. MR3585403

UNITÉ DE RECHERCHE EN MATHÉMATIQUES UNIVERSITÉ DU LUXEMBOURG

MAISON DU NOMBRE

6, AVENUE DE LA FONTE

L-4364 ESCH-SUR-ALZETTE

LUXEMBOURG

E-MAIL: christian.doebler@uni.lu giovanni.peccati@uni.lu 